\title{
Error Estimates for Finite-Element Solutions of Elliptic Boundary Value Problems in Non-Smooth Domains
}

\author{
A.-M. SÄNDIG
}

Es werden Fehlerabschätzungen in verschiedenen Normen (namlich in $W^{m, 2}(\Omega)$ und $L^{p}(\Omega)$, $2 \leqq p \leqq \infty)$ von standarden Finite-Element-Lösungen von elliptischen Randwertproblemen in beschränkten Gebieten im $\mathbb{R}^{N}$ mit konischen Punkten oder nichtüberschneidenden Kanten betrachtet.

Рассматриваются оценки погрешности в различных нормах (а именно в $W^{m, 2}(\Omega)$ " $\left.L^{p}(\Omega), 2 \leqq p \leqq \infty\right)$ от стандартных конечно-әлементных решений әллиптических граничных проблем в ограниченных оп̆ластях в $\mathbb{R}^{N}$ с коническими точками или непересекающимися ребрами.

Error estimates in different norms (namely in $W^{m, 2}(\Omega)$ and $L^{p}(\Omega), 2 \leqq p \leqq \infty$ ) of standard Finite-Element Solutions of elliptic boundary value problems in bounded domains in $\mathbb{R}^{N}$ with conical points or non-intersecting edges are considered.

\section{Introduction}

Boundary value problems are difficult to treat numerically when they are defined in domains with non-smooth boundaries or when the type of the boundary conditions changes. In this situation standard techniques lose accuracy near the resulting singularities and global pollution takes place. The reason for the appearance of this effect is the lower regularity of the solutions of such problems in comparison with those having smooth boundaries. Therefore we first study the regularity of the solutions of elliptic boundary value problems in domains with conical points or non-intersecting edges in the framework of weighted Sobolev spaces using results of V. A. Kondrat'JEv [5-7] and V.G. Maz'JA and B. A. PlamenevskiJ [9-12]. We formulate under which conditions these regularity results are valid for weak solutions of the boundary value problem, too. We introduce standard "Finite Element" spaces and prove error estimates in different norms, namely in $W^{m, 2}(\Omega)$ and $L^{p}(\Omega), 2 \leqq p \leqq \infty$, using essentially the above-mentioned regularity results for the weak solutions of the boundary value problems. The investigation of non-symmetric bilinear forms is included. Results of H. BLum [1] and M. DoBrowolski [3] are special cases.

\section{The boundary value problems}

1.1 The domains. (i) An infinite cone $K \subset \mathbb{R}^{N}$ with the vertex $O$ is defined by its surface equation

$$
x_{N}^{2 p}=\sum_{i_{1}+\ldots+i_{N-1}=2 p} a_{i_{1} \cdots i_{N-1}} x_{1}^{i_{1}} \ldots x_{N-1}^{t_{N-1}}+q(x),
$$

where $\Sigma \cdots \geqq 0, q$ is a smooth function such that $|q(x)|=o\left(\left(x_{1}^{2}+\cdots+x_{N-1}^{2}\right)^{p}\right)$, $p>0$ and $x=\left(x_{1} \cdots x_{N}\right)$. 
(ii) Let $\Omega$ be an open subset of $\mathbb{R}^{N}$ with the compact closure $\bar{\Omega}$ whose boundary $c \Omega$ is an $(N-1)$-dimensional manifold. $0 \in \lambda \Omega$ is a conical point if there is a neighbourhood $U_{\varepsilon}(0)$ of 0 such that $U_{\varepsilon}(0) \cap \Omega$ is diffeomorphic to a cone $K$ intersected with the unit ball. The intersection of $K$ with the unit sphere is a domain $G$ with a smooth boundary $\partial G$. If $N=2$, then the conical points are corner points (with the angle $\left.\omega_{0} \neq \pi\right)$.

(iii) $D \subset \mathbb{R}^{N}, N \geqq 3$, is a dihedral angle if $D=K \times \mathbb{R}^{N-2}$, where $K=\left\{y=\left(y_{1}, y_{2}\right)\right.$ $\left.=(r \cos \omega, r \sin \omega) \in \mathbb{R}^{2}: 0<r<\infty, 0<\omega<\omega_{0}\right\}$ is an infinite cone with the sides $\gamma^{+}=\left\{y \in \mathbb{R}^{2}: \omega=0\right\}$ and $\gamma^{-}=\left\{y \dot{\epsilon} \mathbb{R}^{2}: \omega=\omega_{0}\right\}$. The faces of $D$ are $\Gamma^{ \pm}$ $=\gamma^{ \pm} \times \mathbb{R}^{N-2}$ and the edge of $D$ is $M_{D}=\{(0,0)\} \times \mathbb{R}^{N-2}$.

(iv) Let $\Omega \subset \mathbb{R}^{N}, N \geqq 3$, be a bounded domain with the $(N-1)$-dimensional boundary $\partial \Omega$. $\Omega$ is a domain with non-intersecting $(N-2)$-dimensional edges $M_{1}, \ldots, M_{T-1} \subset \partial \Omega$ if $\boldsymbol{M}=M_{1} \cup \cdots \cup M_{T-1}$ divides $\partial \Omega$ in smooth disjoint connected components $\Gamma_{1}, \ldots, \Gamma_{T}$ (the faces) such that $\delta \Omega=M \cup \Gamma_{1} \cup \cdots \cup \Gamma_{T}$ and if there is a neighbourhood of each point of $M$ in which $\Omega$ is diffeomorphic to an $N$-dimensional dihedral angle $D$.

1.2 The differential operators. We consider the linear differential operators

and

$$
A\left(x, D_{x}\right)=\sum_{\left|y_{1},\right| \beta \mid \leqq m}(-1)^{|\eta|} \dot{c}^{\gamma}\left(a_{\gamma \beta}(x) \delta^{\beta}\right)=\sum_{|\alpha| \leqq 2 m} a_{\alpha}(x) D_{x}^{\alpha}
$$

$$
B,\left(x, D_{x}\right)=\sum_{|\alpha| \leqq m_{j}} b_{j, \alpha}(x) D_{x}^{\alpha} \quad(x \in \partial \Omega \backslash M ; j=1, \ldots, m),
$$

where $M$ is the set of conical points or the set of edges. We denote

$$
D_{x}^{\alpha}=(-\mathrm{i})^{|\alpha|} \frac{\partial^{\alpha_{1}+\cdots+\alpha_{N}}}{\partial x_{1}^{\alpha_{1}} \cdots \partial x_{N}{ }^{\alpha_{N}}}=(-\mathrm{i})^{|\alpha|} \tilde{\sigma}^{\alpha}, \quad \alpha=\left(\alpha_{1}, \ldots, \alpha_{N}\right) \in N_{0}{ }^{N} .
$$

Assume that the coefficients of $A$ are smooth in $\bar{\Omega}$ and those of $B_{j}$ are smooth on the sides or faces of $\partial \Omega$. If $N=2$, the change of the type of the boundary conditions is admissible, also for $\omega_{0}=\pi$. If $N \geqq 3$ and $\Omega$ is a domain with non-intersecting edges, we write instead of (1.2)

$$
B_{j}^{(q)}\left(x, D_{x}\right)=\sum_{|\alpha| \leqq m_{j, q}} b_{j, \alpha}^{(q)}(x) D_{x}^{\alpha} \quad\left(x \in \Gamma_{q} ; q=1, \ldots, T\right),
$$

where the coefficients $b_{j, a}^{(q)}$ are smooth on $\bar{\Gamma}_{q}$. Assume that $A$ is elliptic and $\left\{B_{1}, \ldots, B_{m}\right\}$ or $\left\{B_{1}{ }^{(q)}, \ldots, B_{m}^{(q)}\right\}_{q=1, \ldots .}$ are normal systems on $\partial \Omega \backslash M$ which cover $A$. We denote by

$$
\begin{aligned}
& \mathfrak{U}\left(x, D_{x}\right)=\left\{A\left(x, D_{x}\right), B_{1}\left(x, D_{x}\right), \ldots, B_{m}\left(x, D_{x}\right)\right\}, \\
& \mathfrak{A}\left(x, D_{x}\right)=\left\{A\left(x, D_{x}\right), B_{1}^{(q)}\left(x, D_{x}\right), \ldots, B_{m}^{(q)}\left(x, D_{x}\right)\right\}_{q=1 \ldots .} T
\end{aligned}
$$

the operators defined by (1.1) and (1.2) or by (1.1) and (1.2'), respectively.

1.3 The spaces. We introduce the spaces mapping by the operators $\mathfrak{x}\left(x, D_{x}\right)$.

(i) The space $V^{k, p}(\Omega, \beta(\cdot))$ is the closure of the set $C_{M}{ }^{\infty}(\Omega)=\left\{u \in C^{\infty}(\bar{\Omega}): \operatorname{supp} u \cap M\right.$ $=\varnothing\}$ with respect to the norm

$$
\left\|u ; V^{k, p}(\Omega, \beta(\cdot))\right\| \doteq\left(\sum_{|\alpha| \leqq k} \int_{\Omega} r^{p(\beta(\zeta)-k+|\alpha|)}\left|D^{\alpha} u\right|^{p} d x\right)^{1 / p},
$$

wh ere $r=r(x)=\operatorname{dist}(x, M)=|x-\zeta|, \zeta \in M<\partial \Omega, \beta=\beta(\cdot)$ is a smooth function defined on $M$. $\zeta$ is uniquely determined if $x$ is sufficiently close to $M, \beta=\beta(\cdot)$ $=$ const if $M$ consists of one conical point only. 
(ii) Let $\Omega$ be a domain with a single_conical point 0 . Then the factor space

$$
V^{k-1 / p \cdot p}(\partial \Omega, \beta)=V^{k, p}(\Omega, \beta) / V_{0}{ }^{k} p(\Omega, \beta)
$$

is the space of traces, where $V_{0}^{k, p}(\Omega, \beta)$ is the closure of the set $C_{\partial \Omega}^{\infty}(\Omega)$ with respect to the norm (1.4). Let $\Omega$ be a domain with non-intersecting edges and the faces $\Gamma_{q}$. The space of traces on $\Gamma_{q}$ of functions from $V^{k, p}(\Omega, \beta(\cdot))$ is the factor space

$$
V^{k-1 / p, p}\left(\Gamma_{q}, \beta(\cdot)\right)=V^{k, p}(\Omega, \beta(\cdot)) / V_{0}^{k, p}\left(\Omega, \Gamma_{q}, \beta(\cdot)\right),
$$

where $V_{0}^{k . p}\left(\Omega, \Gamma_{q}, \beta(\cdot)\right)$ is the closure of $C_{\Gamma_{q}}^{\infty}(\Omega)$ with respect to the norm (1.4).

1.4 The boundary value problems. (i) Let $\mathfrak{A}\left(x, D_{x}\right)$ be given by (1.3) or $\left(1.3^{\prime}\right)$, and let $l \geqq 0$ be an integer. We consider the operators

$$
\mathfrak{A}\left(x, D_{x}\right): V^{2 m+l, p}(\Omega, \beta) \rightarrow V^{l, p}(\Omega, \beta) \times \prod_{j=1}^{m} V^{2 m+l-m_{j}-1 / p, p}(\hat{c} \Omega, \beta)
$$

or, in the second case,

$$
\mathfrak{A}\left(x, D_{x}\right): V^{2 m+l, p}(\Omega, \beta(\cdot)) \rightarrow V^{l, p}(\Omega, \beta(\cdot)) \times \prod_{q=1}^{T} \prod_{j=1}^{m} V^{2 m+1-m_{j, q}-1 / p, p}\left(\Gamma_{q}, \beta(\cdot)\right) .
$$

That means we consider the "classical" problem: investigate the solvability, uniqueness and regularity of $(j=1, \ldots, m ; q=1, \ldots, T)$

or

$$
A\left(x, D_{x}\right) u(x)=f(x) \text { in } \Omega, \quad B,\left(x, D_{x}\right) u(x)=g,(x) \text { on } \partial \Omega
$$

$$
A\left(x, \mathcal{D}_{x}\right) u(x)=f(x) \text { in } \Omega, \quad B_{j}^{(q)}\left(x, D_{x}\right) u(x)=g_{j}^{(q)}(x) \text { on } \Gamma_{q}
$$

or, especially, of

or

$$
A\left(x, D_{x}\right) u(x)=f(x) \text { in } \Omega, \quad B,\left(x, D_{x}\right) u(x)=0 \text {, on } \partial \Omega
$$

$$
A\left(x, D_{x}\right) u(x)=f(x) \text { in } \Omega, \quad B_{j}^{(q)}\left(x, D_{x}\right) u(x)=0 \text { on } \Gamma_{q}
$$

in the above-mentioned weighted Sobolev spaces.

(ii) Assume that the boundary value problem $(1 . \overline{9})$ or $\left(1.9^{\prime}\right)$ can be formulated as a weak problem: find a solution $u \in V$ such that, for a given $f \in V^{*}$,

$$
a(u, v)=\int_{\Omega} \sum_{|\gamma|,|\beta| \leqq m} a_{\gamma \beta}(x) \hat{c}^{\beta} u \partial^{\gamma} v d x=(f, v) \quad \forall v \in V
$$

and investigate the regularity if $f$ is from a subspace of $V^{*} . V$ is a subspace of $W^{m, 2}(\Omega)$ which is determined by the essential boundary conditions of $(1.9)$ or $\left(1.9^{\prime}\right) ; V^{*}$ is its dual space and the relation (1.10) guarantees that the non-essential boundary conditions are satisfied. $W^{m, 2}(\Omega)$ denotes the usual Soboler space.

\section{Solvability and regularity results}

We formulate without proofs solvability and regularity results of V. A. Kondrat'jev, V. G. Maz'ja and B. A. Plamenevskij. We investigate when these'regularity results are also valid for weak solutions $u$ of $(1.10)$.

2.1 Solvability and regularity results for domains with conical points. Let $\Omega$ be a bounded domain with a single conical point 0 . For simplicity we assume that there is a ball-neighbourhood of 0 where $\Omega$ coincides with the cone $K$. 
(i) First we consider a special boundary value problem in $K$, which is generated by the principal parts of the operators (1.1) and (1.2) with frozen coefficients at $0(j=1$, $\ldots, m)$ :

$$
\begin{array}{ll}
A_{0}\left(0, D_{x}\right) u(x)=\sum_{|x|=2 m} a_{\alpha}(0) D^{\alpha} u(x)=f(x) & \text { in } K, \\
B_{0, j}\left(0, D_{x}\right) u(x)=\sum_{|\alpha|=m_{j}} b_{j, \alpha}(0) D^{\alpha} u(x)=g_{j}(x) & \text { on } \partial K .
\end{array}
$$

Introducing polar coordinates $(r, \omega)$, setting $r=\mathrm{e}^{\tau}$ and using the complex Fourier transform $\int_{-\infty}^{+\infty} \mathrm{e}^{-\mathrm{i} \lambda \tau} f(\tau) d \tau=\tilde{f}(\lambda), \lambda \in \mathbb{C}$, we obtain a boundary value problem with parameter $\lambda$ in the domain $G=\Omega \cap$ "sphere of the ball-neighbourhood":

$$
\begin{array}{ll}
L\left(\omega, D_{\omega}, \lambda\right) \tilde{u}(\lambda, \omega)=\tilde{F}(\lambda, \omega) & \text { for } \omega \in G, \\
M,\left(\omega, D_{\omega}, \lambda\right) \tilde{u}(\lambda, \omega)=\tilde{G}_{j}(\lambda, \omega) & \text { for } \omega \in \partial G(j=1, \ldots, m),
\end{array}
$$

where $A_{0}\left(0, D_{x}\right)=r^{-2 m} L\left(\omega, D_{\omega}, r D_{r}\right), B_{0,1}\left(0, D_{x}\right)=r^{-m_{j}} M_{,}\left(\omega, D_{\omega}, r D_{r}\right)$. The corresponding operator $\mathfrak{A}_{0}(\lambda)=\left\{L\left(\omega, D_{\omega}, \lambda\right), M_{,}\left(\omega, D_{\omega}, \lambda\right)\right\}_{j=1, \ldots, m}$ maps $W^{2 m+l, p}(G)$ into $W^{l, p}(G) \times \prod_{j=1}^{m} W^{2 m-l-m_{j}-1 / p, p}(G)$.

(ii) The distribution of the generalized eigenvalues of $\mathfrak{A}_{0}(\lambda)$ plays an important role both for the solvability and for the regularity of problem (1.8). The following theorems were proved by V. A. Koxprat'Jev [5: Theorem 3.2] for $p=2$ and by V. G. Maz'JA and B. A. PlamenevskiJ [10: Theorem 6.1] for $p \neq 2$.

Theorem 1: $\mathfrak{A}\left(x, D_{x}\right)$ defined by $(1.7)$ is a Fredholm operator iff no eigenvalue of $\mathfrak{U}_{0}(\lambda)$ lies on the line $\operatorname{Im} \lambda=\beta+N / p-2 m-l$.

Theorem 2: If no eigenvalue of $\mathfrak{A}_{0}\left(\hat{\lambda}_{0}\right)$ is situated in the strip $\beta_{1}+N / p_{1}-2 m-l_{1}$ $\leqq \operatorname{Im} \lambda \leqq \beta+N / p-2 m-l$, then the solution $u \in V^{l+2 m, p}(\Omega, \beta)$ of $(1.9)$ is contained in $\dot{V}^{l_{1}+2 m, p_{1}}\left(\Omega, \beta_{1}\right)$, too, provided $f \in V^{l, p}(\Omega, \beta) \cap V^{l_{1}, p_{1}}\left(\Omega, \beta_{1}\right)$. Here $l$ and $l_{1}$ are nonnegative integers.

If eigenvalues $\lambda_{j}$ of $\mathfrak{U}_{0}\left(\hat{\lambda}_{1}\right)$ lie in the strip $h_{1}=\beta_{1}+N / p_{1}-2 m-l_{1}<\operatorname{Im} \lambda<\beta$ $+N / p-2 m-l=h$ (but not on the lines $\operatorname{Im} \lambda=h, \operatorname{Im} \lambda=h_{1}$ ), then the following expansion holds near 0 :

$$
\eta u=\sum_{h_{1}<\operatorname{Im} \lambda_{j}<h} \sum_{\mu=0}^{\left[h-h_{1}\right]} r^{\mathrm{i} \lambda+\mu P_{j \mu}}(\log r)+w,
$$

where $\eta$ is an appropriate cut-off function, $\eta w \in V^{l_{1}+2 m, p_{1}}\left(\Omega, \beta_{1}\right),\left[h-h_{1}\right]$ is the biggest integer which is less than $h-h_{1}, P_{j \mu}$ are polynomial functions with coefficients depending on $\omega$.

2.2 Solvability and regularity results for domains with edges. Let $\Omega$ be a bounded domain with the only edge $M$. We consider a point $z_{0} \in M$ and assume again for simplicity that $\Omega$ coincides in a ball-neighbourhood of $z_{0}$ with a dihedral angle $D=K \times M$. We take in this neighbourhood the coordinate system $x=(y, z)$ $=\left(y_{1}, y_{2}, z_{1}, \ldots, z_{N-2}\right), y \perp z, y \in K, z \in M$.

(i) We start with a special boundary value problem in $D$, which is generated by the principal parts of the operators $A$ and $B_{j}^{(q)}$ defined by (1.1) and $\left(1.2^{\prime}\right), q=1,2$ :

$$
\begin{aligned}
A_{0}\left(z_{0}, D_{x}\right) u(x) & =A_{0}\left(z_{0}, D_{\nu} D_{z}\right) u(x) \\
& =\sum_{\substack{|x|=2 m \\
\alpha_{1}+\alpha_{z}=\alpha}} a_{\alpha}\left(z_{0}\right) D_{y}{ }^{\alpha_{1}} D_{z}{ }^{\alpha_{2}} u(x)=f(x) \text { in } D,
\end{aligned}
$$




$$
\begin{aligned}
B_{0, j}^{ \pm}\left(z_{0}, D_{x}\right) u(x) & =B_{0, j}^{ \pm}\left(z_{0}, D_{\nu} D_{z}\right) u(x) \\
& =\sum_{\substack{|\alpha|=m_{j} \pm \\
\alpha_{1}+\alpha_{2}=\alpha}} b_{j \alpha}^{ \pm}\left(z_{0}\right) D_{\nu}^{\alpha_{1}} D_{z}^{\alpha_{3}} u(x)=g_{j}^{ \pm}(x) \text { on } \Gamma=.
\end{aligned}
$$

Here \pm is used instead of $q=1,2, \Gamma^{ \pm}$are the faces of $D, j=1, \ldots, m$. After appropriate transformations we get a two-dimensional boundary value problem with parameters in the cone $K$, namely

$$
\begin{array}{ll}
A_{0}\left(z_{0}, D_{y}, \theta\right) \bar{u}=\bar{f} & \text { in } K, \\
B_{0, j}^{ \pm}\left(z_{0}, D_{y}, \theta\right) \tilde{u}=\bar{g}, \pm & \text { on } \partial K, \theta \in S^{N-3},
\end{array}
$$

where $S^{N-3}$ is the sphere of the unit ball in $\mathbb{R}^{N-2}$. We denote

where

$$
\begin{aligned}
& \mathfrak{A}_{0}\left(z_{0}, \theta\right)^{\bullet}=\left\{A_{0}\left(z_{0}, D_{y}, \theta\right), B_{0, j}^{ \pm}\left(z_{0}, D_{\nu}, \theta\right)\right\}_{j=1, \ldots, m}, \\
& \mathfrak{A}_{0}\left(z_{0}, \hat{\imath}\right)=\left\{L\left(\omega, D_{\omega} \hat{\imath}\right), M_{j}^{\ddagger}\left(\omega, D_{\omega}, \lambda_{\imath}\right)\right\}_{\gamma=1, \ldots, m},
\end{aligned}
$$

$$
\begin{aligned}
& A_{0}\left(z_{0}, D_{\nu}, 0\right)=r_{z_{0}}^{-2 m} L\left(\omega, D_{\omega}, r_{z_{0}} D r_{z_{0}}\right), \\
& B_{0, j}^{ \pm}\left(z_{0}, D_{\nu}, 0\right)=r_{z_{0}}{ }^{-m_{g}^{ \pm}} M{ }_{1}^{ \pm}\left(\omega, D_{\omega}, r_{z_{0}} D r_{z_{0}}\right)
\end{aligned}
$$

and $r_{z_{0}}=\left|x-z_{0}\right|$. We have

$$
\mathfrak{A}_{0}\left(z_{0}, \lambda\right): W^{2 m, 2}\left(G^{\prime}\right) \rightarrow L^{2}(G) \times \mathbb{C}^{m} \times \mathbb{C}^{m},
$$

where $G=\left\{\omega: 0<\omega<\omega_{0}\right\}$, for $l=0, p=2$.

(ii) The following properties of $\mathscr{U}_{0}\left(z_{0}, \theta\right)$ and $\left(\mathscr{U}_{0}\left(z_{0}, \hat{\imath}\right)\right.$ determine the solvability and regularity of problem $\left(1.9^{\prime}\right)$ :

$\left.\begin{array}{l}\text { ker } \mathfrak{A}_{0}\left(z_{0}, \theta\right) \text { and coker } \mathfrak{A}_{0}\left(z_{0}, \theta\right) \text { are trivial for all } \theta \in S^{N-3} \\ \text { and for all } z_{0} \in M_{0} \text {. }\end{array}\right\}$

The line $\operatorname{Im} \lambda\left(z_{0}\right)=\beta\left(z_{0}\right)+1-2 m$ does not contain eigenvalues of $\mathfrak{A}_{0}\left(z_{0}, \lambda\right)$ for all $z_{0} \in M$.

We intwoduce the eigenvalues $\lambda_{-}\left(z_{0}\right)$ and $\lambda_{+}\left(z_{0}\right)$ of $\mathfrak{U}_{0}\left(z_{0}, \lambda_{-}\right)$by the property:

The strip $\operatorname{Im} \lambda_{-}\left(z_{0}\right)<\beta\left(z_{0}\right)+1-2 m<\operatorname{Im} \lambda_{+}\left(z_{0}\right)$ is free of eigen-
values of $\mathfrak{A}_{0}\left(z_{0}, \lambda\right)$.

Theorem 3: The conditions (2.2) and (2.3) are necessary and sufficient that the operator of the problem $\left(1.8^{\prime}\right)$,

$$
\begin{aligned}
\mathfrak{U}\left(x, D_{x}\right): & V^{l+2 m, p}(\Omega, \beta(\cdot)+1-2 / p+l) \\
\rightarrow & V^{l, p}(\Omega, \beta(\cdot)+1-2 / p+l) \times \sum_{q \rightarrow 1}^{2} \prod_{j=1}^{m} V^{l+2 m-m_{j}^{(\ell)}-1 / p, p} \\
& \times\left(\Gamma_{q}, \beta(\cdot)+1-2 / p+l\right),
\end{aligned}
$$

is a Fredholm operator for any $p \in(1, \infty), l=0,1,2, \ldots$

Theorem 4: Let $\beta=\beta(z)$ and $\beta_{1}=\beta_{1}(z)$ be smooth functions defined on the edge $M \subset \partial \Omega$ and let $u \in V^{l+2 m, p}(\Omega, \beta(\cdot)+1-2 / p+l)$ be a solution of problem (1.9), where the right-hand side $f \in V^{l_{1}, p_{1}}\left(\Omega, \beta_{1}(\cdot)+1-2 / p_{1}+l_{1}\right)$. Assume that the conditions (2.2) and (2.3) are valid and that $\operatorname{Im} \lambda_{-}(z)<\beta_{1}(z)+1-2 m<\operatorname{Im} \lambda_{+}(z)$ for all $z \in M$. Then $u \in V^{l_{1}+2 m, p_{1}}\left(\Omega, \beta_{1}(\cdot)+1-2 / p_{1}+l_{1}\right)$, too. 
If eigenvalues $\lambda_{,}(z)$ of $\mathfrak{U}_{0}(z, \lambda)$ lie in the strip $h_{1}(z)=\beta_{1}(z)+1-2 m<\operatorname{Im} \lambda_{\eta}(z)$ $\left\langle\beta(z)+1-2 m=h(z)\right.$ (but not on the lines $\operatorname{Im} \lambda_{=}=h_{1}(z)$ and $\operatorname{Im} \lambda_{2}=h(z)$ ), then an expansion simitar as for conical points holds:

$$
u(x)=\sum_{\gamma \in I} \eta(x) c_{\gamma}(x) u_{\gamma}(x)+w(x),
$$

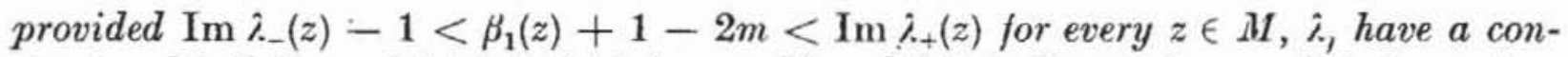
stant multiplicity and $\lambda_{i}(z) \neq \lambda_{,}(z)$ for $z \in M$ and $i \neq j$. Here $\gamma$ is a multiindex, $\eta$ is a cut-off function with $\eta(x)=1$ near $M, u_{y}$ are singular functions, $c_{\gamma}$ are the coefficients and $w \in V^{l_{1}+2 m, p_{1}}\left(\Omega, \beta_{1}(\cdot)+1-2 / p_{1}+l_{1}+\varepsilon\right)$. Here $\varepsilon>0$ is a real number.

2.3 Regularity results for weak solutions in domains with conical points. Let $\Omega \subset \mathbb{R}^{N}$ be a bounded domain with the single conical point $0 \in \partial \Omega$. Assume in the following that the weakly formulated boundary value problem (1.10) has a uniquely defined solution $u \in V \subset W^{m, 2}(\Omega)$, or more precisely, that the Lax-Milgram Theorem holds, i.e.

$$
\begin{array}{ll}
|a(u, v)| \leqq c_{1}\left\|u ; W^{m, 2}(\Omega)\right\|\left\|v ; W^{m, 2}(\Omega)\right\| & \text { for all } u, v \in V, \\
|a(u, u)| \geqq c_{2}\left\|u ; W^{m, 2}(\Omega)\right\|^{2} & \text { for all } u \in V .
\end{array}
$$

In order to be able to use the Regularity Theorem 2 we demand that for certain right-hand sides the solutions $u$ of (1.10) are contained in a reasonable weighted space, namely:

(R) If $f \in L^{2}(\Omega)$, then $u \in V \cap V^{2 m, 2}(\Omega, m)$.

Lemma 1: If the line $\operatorname{Im} \hat{\lambda}=N / 2-m$ is free of eigenvalues of $\mathfrak{A}_{0}(\lambda)$ and $m \leqq N / 2$ or the Dirichlet problem is given, then the property $(\mathrm{R})$ is valid.

Proof: Let

$$
W^{m, 2}(\Omega, \delta)=\left\{f: \| f ; W^{m, 2}(\Omega, \delta)=\left(\sum_{|\alpha| \leqq m} \int_{\Omega} r^{2 \delta}\left|D^{a} f\right|^{2} d x\right)^{1 / 2}<, \infty\right\} .
$$

The following imbeddings hold [4: Corollary 3.1]:

$$
\begin{aligned}
& V \subset W^{m, 2}(\Omega) \subset W^{m, 2}(\Omega, \delta) \text { for } \delta \geqq 0, \\
& W^{m, 2}(\Omega, \delta) \subset L^{2}(\Omega, \delta-m) \text { for } \delta>m-N / 2 .
\end{aligned}
$$

It follows from [5: Theorem 3.3] that for $f \in L^{2}(\Omega)$ (even for $f \in L^{2}(\Omega, \delta+m)$ ) the solution $u$ of $(1.10)$ is contained in $V^{2 m, 2}(\Omega, \delta+m)$. Analogously to (2.4) we introduce the eigenvalues $\lambda_{-}$and $\lambda_{+}$of $\mathfrak{A}_{0}\left(\lambda_{-}\right)$as follows for $\beta=m$ :

The strip $\operatorname{Im} \lambda_{-}<N / 2-m<\operatorname{Im} \lambda_{+}$is frèe of eigenvalues of $\mathfrak{A}_{0}\left(\lambda_{)}\right)$.

We now choose $\delta>0$ so small that $\operatorname{Im} \lambda_{-}<-m+N / 2+\delta<\operatorname{Im} \lambda_{+}$. Theorem 2 yields the assertion for $m \leqq N / 2$. The assertion for the Dirichlet problem follows immediately since $W_{0}^{m .2}(\Omega)=V \subset V^{m, 2}(\Omega, 0) \subset L^{2}(\Omega,-m)$

Remark 1: The condition $m \leqq N / 2$ of Lemma 1 can be weakened by the condition: There is a $\delta \geqq 0$ with $\delta>m-N / 2$ and $-m+N / 2+\delta<\operatorname{Im} \lambda_{+}$.

Lemma 2: Assume that the line $\operatorname{Im} \lambda=-m+N / 2$ is free of eigenvalues of $\mathfrak{A}_{0}(\lambda)$ and the property $(\mathrm{R})$ holds. Then there is a uniquely determined solution $u \in \nabla^{2 m, p}(\Omega$, $\left.\operatorname{Im} \lambda_{-}-N / p+2 m+\varepsilon\right)$ of $(1.10)$ for every $f \in L^{p}\left(\Omega, \operatorname{Im} \lambda_{-}-N / p+2 m+\varepsilon\right)$, 
$1<p<\infty$, where $\varepsilon>0$ is a small real number, and

$$
\begin{aligned}
& \left\|u ; V^{2 m, p}\left(\Omega, \operatorname{Im} \lambda_{-}-N / p+2 m+\varepsilon\right)\right\| \\
& \leqq C\left\|f ; L^{p}\left(\Omega, \operatorname{Im} \lambda_{-}-N / p+2 m+\varepsilon\right)\right\| .
\end{aligned}
$$

Proof: The Regularity Theorem 2 and the Lax-Milgram Theorem imply that for every $f \in L^{2}(\Omega) \cap L^{p}\left(\Omega, \operatorname{Im} \lambda_{-}-N / p+2 m+\varepsilon\right)$ there is a uniquely determined solution $u \in V^{2 m, p}\left(\Omega, \operatorname{Im} \lambda_{-}-N / p+2 m+\varepsilon\right)$ of $(1.10)$. Since $L^{2}(\Omega) \cap L^{p}\left(\Omega, \operatorname{Im} \lambda_{-}\right.$ $-N / p+2 m+\varepsilon)$ is dense in $L^{p}\left(\Omega, \operatorname{Im} \lambda_{-}-N / p+2 m+\varepsilon\right)$ (this follows immediately from the definition of the weighted spaces in Subsection 1.3) and the operator (2.5) is a Fredholm operator we get the assertion

Remark 2: Lemma 2 is valid for all $f \in L^{p}(\Omega)$ provided $\operatorname{Im} \lambda_{-}-N / p+2 m \geqq \cdot 0$. In this case we have $\left\|u ; V^{2 m, p}\left(\Omega, \operatorname{Im} \lambda_{-}-N / p+2 m+\varepsilon\right)\right\| \leqq C\left\|f ; L^{p}(\Omega)\right\|$. However, if $\operatorname{Im} \lambda_{-}-N / p$ $+2 m<0, N / p-2 m<\operatorname{Im} \lambda_{+}$and the suppositions of Lemma 2 are satisfied, then there exists a uniquely determined solution $u \in V^{2 m, p}(\Omega, 0)$ of $(1.10)$ for every $f \in L^{p}(\Omega)$ and $\left\|u ; V^{2 m, p}(\Omega, 0)\right\| \leqq c\left\|f ; L^{p}(\Omega)\right\|$. Indeed, if $\operatorname{Im} \lambda_{-}-N / p+2 m \geqq 0$, then $L^{p}(\Omega) \subset L^{p}\left(\Omega\right.$, Im $\lambda_{-}$ $-N / p+2 m+\varepsilon)$. If $\operatorname{Im} \lambda_{-}-N / p+2 m<0$ and $N / p-2 m<\operatorname{Im} \lambda_{+}$, then the Regularity Theorem 2 and the Lax-Milgram Theorem imply that for every $f \in L^{2}(\Omega) \cap L^{p}(\Omega)$ there is a uniquely determined solution $u \in V^{2 m, p}(\Omega, 0)$ of $(1.10)$. Since $L^{2}(\Omega) \cap L^{p}(\Omega)$ is dense in $L^{p}(\Omega)$ we again use the Fredholm property of the operator (2.5) and get the assertion.

Lemma 3: Assume that the line $\operatorname{Im} \lambda=-m+N / 2$ is, free of eigenvalues of $\mathfrak{A}_{0}(\lambda)$ and that the property $(\mathrm{R})$ holds. If $f \in L^{p}(\Omega)$ and $\operatorname{Im} \lambda_{-}<N / p, 1<p \leqq \infty$, then the solution $u$ of (1.10) is from $L^{p}(\Omega)$.

Proof: Assume that $1<p<\infty$. If $N / p \leqq N / 2-m$, then Rémark 2 works and $u \in V^{2 m . p}(\Omega, 2 m)$ in the worst case. Since $V^{2 m, p}(\Omega, 2 m) \subset L^{p}(\Omega)$ we get the assertion. If $N / p>N / 2-m$, then the classical imbedding theorem yields that $W^{m, 2}(\Omega)$ $\subset L^{p}(\Omega)$. If $p=\infty$ and therefore $\operatorname{Im} \lambda_{-}<0$, then the assertion follows from the asymptotic expansion given in Theorem $\mathbf{2}$

2.4 Regularity results for weak solutions in domains with edges. Let $\Omega \subset \mathbb{R}^{N}$ be a bounded domain with the only edge $M$. Assume again that the weakly formulated boundary value problem (1.10) has a uniquely determined solution $u \in V \subset W^{m, 2}(\Omega)$ for $f \in V^{*}$. Again we need the regularity condition:

(R) If $f \in L^{2}(\Omega)$, then $u \in V \cap V^{2 m, 2}(\Omega, m), \beta(z)=m$ Ior $z \in M$.

Lemma 4: If the line $\operatorname{Im} \lambda=1-m$ is free of eigenvalues of $\mathfrak{A}_{0}(z, \lambda)$-and if there is a real number $\delta$ with $\delta>m-1$ and $-m+1+\delta<\operatorname{Im} \lambda_{+}(z)$ for all $z \in M$ or if the Dirichlet problem is given, then the property $(\mathrm{R})$ is valid. Especially, if $m=1$ and the line $\operatorname{Im} \lambda=1-m$ is free of eigenvalues of $\mathfrak{U}_{0}(z, \lambda)$, then the property $(\mathrm{R})$ is satisfied.

Proof: We first, remark that J. Rossmand [14] proved the following result: if the line $\operatorname{Im} \lambda=1-m$ is free of eigenvalues of $\mathfrak{A}_{0}(z, \lambda)$ and the Gårding inequality is valid for the problem (1.10), then condition (2.2) is satisfied. Since our problem is even coercive, we can use this result. We now employ the same ideas as in the proof of Lemma 1. We have $u \in W^{m, 2}(\Omega) \subset W^{m, 2}(\Omega, \delta) \subset L^{2}(\Omega, \delta-m)$ for $\delta>m-1$ (cf. [4: Corollary 3.1]). From [12: Theorem 10.2] it follows that $u \in V^{2 m, 2}(\Omega, m+\delta)$. Theorem 4 yields that $u \in V^{2 m, 2}(\Omega, m)$. If $m=1$, then we choose $\delta$ sufficiently small

Analogously to Lemma 2 and Remark 2 we are able to prove

Lemma 5: Assume that the line $\operatorname{Im} \lambda=1-m$ is free of eigenvalues of $\mathfrak{A}_{0}(z, \lambda)$ for $z \in M$ and the property $(\mathrm{R})$ holds. Then there is a uniquely determined solution $u \in V^{2 m, p}$ $\left(\Omega, \operatorname{Im} \lambda_{-}(\cdot)-2 / p+2 m+\varepsilon(\cdot)\right)$ of $(1.10)$ for every $f \in L^{p}\left(\Omega, \operatorname{Im} \lambda_{-}(\cdot)-2 / p+2 m\right.$ 
$+\varepsilon(\cdot)), 1<p<\infty$, where $\varepsilon(z)>0$ are small real numbers and

$$
\begin{aligned}
& \left\|u ; V^{2 m, p}\left(\Omega, \operatorname{Im} \lambda_{-}(\cdot)-2 / p+2 m+\varepsilon(\cdot)\right)\right\| \leqq C \| f ; L^{p}\left(\Omega, \operatorname{Im} \lambda_{.-(\cdot)}\right. \\
& -2 / p+2 m+\varepsilon(\cdot)) \| .
\end{aligned}
$$

It is more suitable to work with a weight with a constant exponent in the following. Therefore we introduce:

Let $z_{0} \in M$ be such that $\operatorname{Im} \lambda_{-}(z) \leqq \operatorname{Im} \lambda_{-}\left(z_{0}\right)$ for all $z \in M$. For this we write $\operatorname{Im} \lambda_{-}\left(z_{0}\right)=\operatorname{Im} \lambda_{-}$. We have $\operatorname{Im} \lambda_{-}(z) \leqq \operatorname{Im} \lambda_{-}<1-m<\operatorname{Im} \lambda_{+}(z)$. Consequently the following Lemma $5^{\prime}$ is weaker than Lemma 5 .

Lemma 5': Assume that the line $\operatorname{Im} \lambda=1-m$ is free of eigenvalues of $\mathfrak{A}_{0}(z, \hat{i})$ for $z \in M$ and that the property $(\mathrm{R})$ holds. Then there is a uniquely determined solution $u \in V^{2 m, p}\left(\Omega, \operatorname{Im} \lambda_{-}-2 / p+2 m+\varepsilon\right)$ of $(1.10)$ for every $f \in L^{p}\left(\Omega, \operatorname{Im} \lambda_{-}-2 / p+2 m\right.$ $+\varepsilon), 1<p<\infty$, where $\varepsilon>0$ is a small real number and

$$
\begin{aligned}
& \left\|u ; V^{2 m, p}\left(\Omega, \operatorname{Im} \lambda_{-}-2 / p+2 m+\varepsilon\right)\right\| \\
& \leqq C\left\|f ; L^{p}\left(\Omega, \operatorname{Im} \lambda_{-}-2 / p+2 m+\varepsilon\right)\right\| .
\end{aligned}
$$

Remark 3: Lemma $5^{\prime}$ is valid for all $f \in L^{p}(\Omega)$ provided $\operatorname{Im} \lambda_{-}-2 / p+2 m \geqq 0$. In this case we have $\left\|u ; V^{2 m, p}\left(\Omega, \operatorname{Im} \lambda_{\lrcorner}-2 / p+2 m+\varepsilon\right) \leqq C\right\| f ; L^{p}(\Omega) \|$. However, if $\operatorname{Im} \lambda_{-}-2 / p$ $+2 m<0$ and the suppositions of Lemma $5^{\prime}$ are satisfied, then there exists a uniquely determined solution $u \in V^{2 m, p}(\Omega, 0)$ of $(1.10)$ for every $f \in L^{p}(\Omega)$ and $\left\|u ; V^{2 m, p}(\Omega, 0)\right\| \leqq C\left\|f ; L^{p}(\Omega)\right\|$.

Lemma 6: Assume that the line $\operatorname{Im} \lambda=1-m$ is free of eigenvalues of $\mathfrak{A}_{0}(z, \lambda)$ for all $z \in M$ and that the property $(\mathrm{R})$ holds. If $f \in L^{p}(\Omega)$, then $u \in L^{p}(\Omega)$ for $1<p<\infty$.

Proof: Since $\operatorname{Im} \lambda_{-}<2 / p$ and the assumptions of Remark 3 are satisfied, we get $u \in V^{2 m, p}\left(\Omega, \operatorname{Im} \lambda_{-}-2 / p+2 m+\varepsilon\right) \subset V^{2 m, p}(\Omega, 2 m) \subset L^{p}(\Omega)$ provided $\operatorname{Im} \lambda_{-}-2 / p$ $+2 m \geqq 0$. In the other case we have $u \in V^{2 m, p}(\Omega, 0) \subset L^{p}(\Omega)$

\section{Finite element methods in domains with eonical points}

3.1 Finite-element spaces. We shortly characterize the finite-element spaces which we use in the following (not only for domains with conical points). Let us consider a family of appropriate partitions $\pi_{h}$ of the bounded domain $\Omega \subset \mathbb{R}^{N}$ depending on the mesh size $h, \bar{\Omega}=\cup\left\{\Omega_{e, h}: e=1, \ldots, E_{h}\right\}, \Omega_{e, h} \in \pi_{h}$. Assume the partition to be uniformly near the set $M \subset \partial \Omega$ in the sense that there is a set $U(h)=\left\{\Omega_{e, h}: e \in J_{h}\right\}$, $\Omega=U(h) \cup(\Omega \backslash U(h))$, such that

$$
M \subset \overline{U(h)}, \quad \operatorname{dist}(x, M)\left\{\begin{array}{l}
\leqq c_{1} h \text { for } x \in U(h), \\
\geqq c_{2} h \text { for } x \in \Omega \backslash U(h),
\end{array}\right.
$$

where the constants $c_{1}$ and $c_{2}$ are independent of $h, J_{h}$ is an index set (see Fig. 1 for conical points and Fig. 2 for an edge).

Let $\left\{S_{h}\right\}$ be a family of finite-dimensional spaces, $S_{h} \subset V$, with the following properties:

$1^{\circ}$ Local approximation: for every $u \in W^{m, p}(\Omega)$ and for all $\Omega_{e, h} \in \tau_{h}$ there is an element $I_{h} u \in S_{h}$ with

$$
\left\|u-I_{h} u ; W^{l, q}\left(\Omega_{e, h}\right)\right\| \leqq C h^{m-l-N(1 / p-1 / q)}\left\|\nabla_{m} u ; L^{p}\left(\Omega_{e, h}\right)\right\|,
$$

where $W^{m, p}(\Omega) \subset W^{l, q}(\Omega), 0 \leqq l \leqq m$ and $1 \leqq p, q \leqq \infty$. 


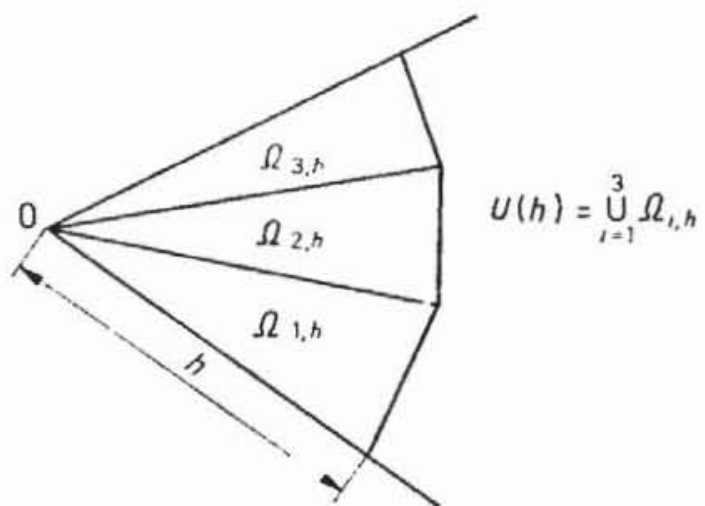

Fig. 1

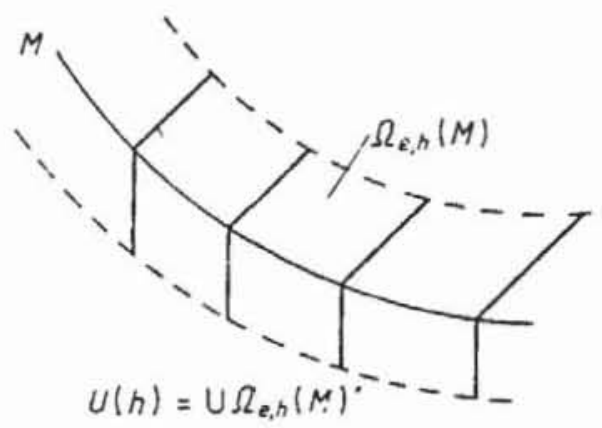

Fig. 2

$2^{\circ}$ Inverse inequality: for all $u_{h} \in S_{h} \subset W^{l, q}(\Omega)$ and $\Omega_{e, h} \in \pi_{h}$

$$
\left\|\nabla_{l} u_{h} ; L^{q}\left(\Omega_{e, h}\right)\right\| \leqq C h^{N\left(1 / q-1 / q_{1}\right)}\left\|\nabla_{l} u_{h} ; L^{q_{1}}\left(\Omega_{e, h}\right)\right\|, \quad 1 \leqq q_{1} \leqq q \leqq \infty,
$$

where $\nabla_{l}$ denotes the field of all derivatives of the order $l$. We refer to [2: Chap. 3], where these properties are considered. The local properties $1^{\circ}$ and $2^{\circ}$ imply global properties (see [8: Theorem 16.7]), namely

$$
\begin{array}{ll}
\left\|u-I_{h} u ; W^{l, p}(\Omega)\right\| \leqq c h^{m-1}\left\|\nabla_{m} u ; L^{p}(\Omega)\right\| & \text { for } p=q, \\
\left\|u-I_{h} u ; W^{l, \infty}(\Omega)\right\| \leqq c h^{m-l-N / p}\left\|\nabla_{m} u ; L^{p}(\Omega)\right\| & \text { for } q=\infty, \\
\left\|\nabla_{l} u_{h} ; L^{p}(\Omega)\right\| \leqq c h^{N\left(1 / q-1 / q_{1}\right)}\left\|\nabla_{l} u_{h} ; L^{q_{1}}(\Omega)\right\| & \text { for } q_{1} \leqq q, u_{h} \in S_{h} .
\end{array}
$$

Definition 1: $P_{h} u \in S_{h} \subset V$ is the finite-element solution of the problem (1.10) if $a\left(P_{h} u, v\right)=(f, v)$ for all $v \in S_{h}$.

Since the Lax-Milgram Theorem is valid, Cea's lemma implies

$$
\left\|u-P_{h} u ; W^{m, 2}(\Omega)\right\| \leqq C \inf _{u_{h} \in S_{h}}\left\|u-u_{h} ; W^{m, 2}(\Omega)\right\| .
$$

3.2 Error estimates in $\boldsymbol{W}^{m, 2}(\Omega)$. The local approximation property $1^{\circ}$ and the estimate (3.5) yield $\left\|u-P_{h} u ; W^{m, 2}(\Omega)\right\|=O\left(h^{m}\right)$ for $u \in W^{2 m, 2}(\Omega)$. If $u \notin W^{2 m, 2}(\Omega)$, then we cannot expect such an estimate in general. Therefore let us characterize those conical or boundary points where the type of those boundary conditions changes which do not imply that $u \in W^{2 m, 2}(\Omega)$.

Definition 2: The point $0_{j}$ from $M$ is significantly singular if

$$
N / 2-2 m<\operatorname{Im} \lambda_{-}{ }^{j}
$$

$\left(\lambda_{-}{ }^{j}\right.$ is defined analogously to $\lambda_{-}$by $\left.(2.8)\right)$.

If this is valid and the assumptions of Lemma 2 are satisfied, we get: if $f \in L^{2}(\Omega)$, then the solution $u$ of the problem $(1.10)$ is from $V^{2 m, 2}\left(\Omega, \operatorname{Im} \lambda_{m}^{j}-N / 2+2 m+\varepsilon\right)$ but not, in general, from $V^{2 m, 2}(\Omega, 0) \subset W^{2 m, 2}(\Omega)$.

Example 1: We consider the Dirichlet problem $\Delta u=-f$ in $\Omega, u=0$ on $\partial \Omega$, where $\Omega$ is a plane domain with the single conical point 0 with the angle $\omega_{0}$. Since $\lambda_{-}=-\pi / \omega_{0} \mathrm{i}$, we have: if $-1<-\pi / \omega_{0}$, which means $\omega_{0}>\pi$, then 0 is a significant singularity.

Theorem 5: Let $a(\cdot, \cdot)$ be a real bilinear form which satisfies the conditions of the Lax-Milgram Theorem (2.6) and (2.7). Assume that there is only one significant singularity $0 \in \partial \Omega$, that the line $\operatorname{Im} \lambda=N / 2-m$ is free of eigenvalues of $\mathfrak{A}_{0}\left(\lambda_{.}\right)$and that the 
property $(\mathrm{R})$ is valid. Let $\left\{S_{h}\right\}$ be a family of finite-element spaces with the properties $1^{\circ}$ and $2^{\circ}$. Then the finite-element solution $P_{h} u \in S_{h}$ approximates the solution $u$ of (1.10) in the sense

$$
\left\|u-P_{h} u ; W^{m, 2}(\Omega)\right\| \leqq C h^{-\operatorname{Im} \lambda_{-}-m+N / 2-\varepsilon}\left\|f ; L^{2}(\Omega)\right\| .
$$

Proof: We use the estimate (3.5) and get

$$
\begin{aligned}
\left\|u-P_{h} ; W^{m, 2}(\Omega)\right\|^{2} \leqq & C\left\|u-I_{h} u ; W^{m, 2}(\Omega)\right\|^{2} \\
\leqq & C\left(\left\|u-I_{h} u ; W^{m, 2}(U(h))\right\|^{2}+\| u-I_{h} u ; W^{m, 2}\right. \\
& \left.\times(\Omega \backslash U(h)) \|^{2}\right),
\end{aligned}
$$

where $I_{h} u$ is defined by $1^{\circ}$ and $U(h)$ was introduced by (3.1). Property $1^{\circ}$, Lemma 2 and (3.1) yield for the first term

$$
\begin{aligned}
& \left\|u-I_{h} u ; W^{m, 2}(U(h))\right\|^{2} \\
& \leqq C\left\|\nabla_{m} u ; L^{2}(U(h))\right\|^{2}=C \sum_{|\alpha|=m} \int_{U(h)} r^{2\left(\operatorname{Im} \lambda_{-}-N / 2+m+\varepsilon-\operatorname{Im} \lambda_{-}+N / 2-m-\varepsilon\right)}\left|D^{\alpha} u\right|^{2} d x \\
& \leqq C\left(c_{1} h\right)^{2\left(-\operatorname{Im} \lambda_{-}+N / 2-m-\varepsilon\right)} \sum_{|\alpha|=m} \int_{U(h)} r^{2\left(\operatorname{Im} \lambda_{-}-N / 2+m+\varepsilon\right)}\left|D^{\alpha} u\right|^{2} d x \\
& \leqq \tilde{C} h^{2\left(-\operatorname{Im} \lambda_{-}+N / 2-m-\varepsilon\right)}\left\|u ; V^{m, 2}\left(\Omega, \operatorname{Im} \lambda_{-}-N / 2+m+\varepsilon\right)\right\|^{2} \\
& \leqq \cdots\left\|u ; V^{2 m, 2}\left(\Omega, \operatorname{Im} \lambda_{-}-N / 2+2 m+\varepsilon\right)\right\|^{2} \\
& \leqq \cdots\left\|f ; L^{2}\left(\Omega, \operatorname{Im} \lambda_{-}-N / 2+2 m+\varepsilon\right)\right\|^{2} \\
& \leqq \widetilde{C} h^{2\left(-\operatorname{Im} \lambda_{-}+N / 2-m-\varepsilon\right)}\left\|f ; L^{2}(\Omega)\right\|^{2} .
\end{aligned}
$$

Further we have for the second term, using again the estimate (2.9),

$$
\begin{aligned}
& \left\|u-I_{h} u ; W^{m, 2}(\Omega \backslash U(h))\right\|^{2} \\
& \leqq C h^{2 m}\left\|\nabla_{2 m} u ; L^{2}(\Omega \backslash U(h))\right\|^{2} \\
& \leqq C h^{2 m} \sum_{|\alpha|=2 m} \int_{\Omega \backslash U(h)} h^{2\left(-\operatorname{Im} \lambda_{-}+N / 2-2 m-\varepsilon\right)+2\left(\operatorname{Im} \lambda_{-}-N / 2+2 m+\varepsilon\right)}\left|D^{\alpha} u\right|^{2} d x \\
& \leqq C\left(1 / C_{2}\right)^{2\left(\operatorname{Im} \lambda_{-}-N / 2+2 m+z\right)} h^{2 m+2\left(-\operatorname{Im} \lambda_{-}+N / 2-2 m-\varepsilon\right)} \\
& \times \sum_{|\alpha|=2 m} \int_{\Omega \backslash U(h)} r^{2\left(\operatorname{Im} \lambda_{-}-N / 2+2 m+\varepsilon\right)}\left|D^{a} u\right|^{2} d x \\
& \leqq C_{3} h^{\left.21-\operatorname{Im} L_{-}+N / 2-m-\varepsilon\right)}\left\|u ; V^{2 m, 2}\left(\Omega, \operatorname{Im} \lambda_{-}-N / 2+2 m+\varepsilon\right)\right\|^{2} \\
& \leqq G_{4} h^{2\left(-\operatorname{Im} 2_{-}+N / 2-m-\varepsilon\right)}\left\|f ; L^{2}(\Omega)\right\|^{2} \text {. }
\end{aligned}
$$

Both estimates imply the assertion (3.7)

3.3 The Aubin-Nitsche trick. The Aubin-Nitsche trick allows to estimate the error $u-P_{\Lambda} u$ in $L^{p}(\Omega), 2 \leqq p \leqq \infty$. The essential idea is to introduce the adjoint problem to the weak problem (1.10).

Definition 3: Let $a(\cdot, \cdot)$ be a real bilinear form defined on $V \times V$. The adjoint problem is: Find a solution $u \in V$ of $(1.10)$ such that $a^{*}(u, v)=a(v, u)=g(v)$ for all $v \in V, g \in V^{*}$. 
We need in the following the regularity of the solution of the adjoint problem. Therefore we shortly formulate some results of V. G. Maz'JA and B. A. PlamenevskiJ [10: §3] without proofs, first for domains with a conical point 0 .

Lemma 7 : Lét $\mathfrak{2}_{0}^{*}(\mu)$ be the operator determined by $a^{*}(\cdot, \cdot)$ and defined analogously to $\mathfrak{U}_{0}(\hat{\lambda})$. Then $\lambda_{0}$ is an eigenvalue of $\mathfrak{U}_{0}(\lambda)$ if and only if $\mu_{0}=\hat{\lambda}_{0}+\mathrm{i}(-2 m+N)$ is an eigenvalue of $\mathfrak{A}_{0}^{*}(\mu)$.

Corollaries: (i) If the line $\operatorname{Im} \lambda=-m+N / 2$ is free of eigenvalues of $\mathfrak{A}_{0}(\lambda)$, then it is free of eigenvalues of $\mathfrak{A}_{0} *(\mu)$, too. (ii) The imaginary parts of the eigenvalues of $\mathfrak{U}_{0}(\lambda)$ and $\mathfrak{A}_{0} *(\mu)$ are situated symmetrically to the point $-m+N / 2$ :

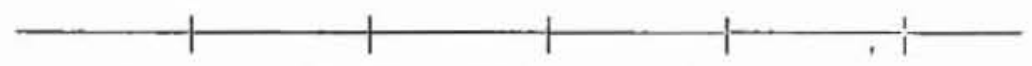

$\operatorname{Im} \mu_{-} \quad \operatorname{Im} \lambda_{-} \quad N / 2-m \operatorname{Im} \mu_{+} \operatorname{Im} \lambda_{+} \quad$ Fig. 3

(iii) Let $\lambda_{-}$and $\lambda_{+}$be those eigenvalues of $\mathfrak{U}_{0}\left(\lambda_{2}\right)$ which are defined by (2.8). The corresponding eigenvalues of $\mathfrak{Q}_{0}^{*}(\mu)$ are $\mu_{-}^{*}=\bar{\lambda}_{+}+(-2 m+N)$ i and $\mu_{+}=\bar{\lambda}_{-}+\mathrm{i}(-2 m+N)$ and consequently $\operatorname{Im} \mu_{-}=-\operatorname{Im} \lambda_{+}-2 m+N$ and $\operatorname{Im} \mu_{+}=-\operatorname{Im} \lambda_{-}-2 m+N$.

Lemma 8: Let $X$ be a Banach space with $V \subset X$ and $X_{1}, X_{2}$ a pair of Banach spaces with $|a(u, v)| \leqq C\left\|u ; X_{1}\right\|\left\|v ; X_{2}\right\|$ for all $u, v \in V$ and $S_{h} \subset X_{1} \cap X_{2}$. Then the finiteelement solution $P_{h} u$ approximates the solution $u$ of the problem (1.10) in $X$ in the sense

$$
\left\|u-P_{h} u ; X\right\| \leqq C\left\|u-P_{h} u ; X_{1}\right\| \sup _{0 \neq g \in X^{*}}\left\{\left\|u_{g}-I_{h} u_{g} ; X_{2}\right\| /\left\|g ; X^{*}\right\|\right\},
$$

where $a^{*}\left(u_{g}, v\right)=a\left(v, u_{g}\right)=(g, v)$ for $g \in X^{*}$ and $v \in V, X^{*}$ is the dual space of $X$.

Proof: We have

$$
\begin{aligned}
\left\|u-P_{h} u ; X\right\| & =\left\|u-P_{h} u ; X^{* *}\right\|=\sup _{\left\|g ; X^{*}\right\|=1}\left|\left(g, u-P_{h} u\right)\right| \\
& =\sup _{0 \neq g \in X^{*}}\left\{\left|\left(g, u-P_{h} u\right)\right| /\left\|g ; X^{*}\right\|\right\} \\
& =\sup _{0 \neq g \in X^{*}}\left\{\left|a\left(u-P_{h} u, u_{g}\right)\right| \cdot\left\|g ; X^{*}\right\|\right\} \\
& =\sup _{0 \neq g \in X^{*}}\left\{\left|a\left(u-P_{h} u, u_{g}-I_{h} u_{g}\right)\right| /\left\|g ; X^{*}\right\|\right\} \\
& \leqq C\left\|u-P_{h} u, X_{1}\right\| \sup _{0 \neq g \in X^{*}}\left\{\left\|u_{g}-I_{h} u_{g} ; X_{2}\right\| /\left\|g ; X^{*}\right\|\right\}
\end{aligned}
$$

Example 2: For $X=L^{2}(\Omega), X_{1}=W^{m, 2}(\Omega)=X_{2}$ we get for the solution $u$ of $(1.10)$

$$
\begin{aligned}
& \left\|u-P_{h} u ; L^{2}(\Omega)\right\| \\
& \leqq C\left\|u-P_{h} u ; W^{m, 2}(\Omega)\right\| \sup _{0 \neq g \in L^{2}(\Omega)}\left\{\left\|u_{\sigma}-I_{h} u_{g} ; W^{m \cdot 2}(\Omega)\right\| /\left\|g ; L^{2}(\Omega)\right\|\right\} .
\end{aligned}
$$

Assume that the assumptions of Theorem 5 are satisfied and that $(\mathrm{R})$ is valid for $a^{*}(u, v)$. The estimate (3.7) for $u$ and the estimates (3.8) and (3.9) for $u_{g}$ yield

$$
\left\|u-P_{h} u ; L^{2}(\Omega)\right\| \leqq C h-\operatorname{Im} \lambda_{-}+N / 2-m_{-} \varepsilon-\operatorname{Im} \mu_{-}+N / 2-m-\varepsilon\left\|f ; L^{2}(\Omega)\right\|
$$

provided Im $\mu_{-}-N / 2+2 m \geqq 0$. From Corollary (iii) follows

$$
\left\|u-P_{h} u ; L^{2}(\Omega)\right\| \leqq C h-\operatorname{Im} \lambda_{-}+\operatorname{Im} \lambda_{+}-8\left\|f ; L^{2}(\Omega)\right\| \text { for }-\operatorname{Im} \lambda_{+}+N / 2 \geqq 0 .
$$

If $-\operatorname{Im} \lambda_{+}+N / 2<0$, then it follows from Remark 2 that $u_{g} \in V^{2 m \cdot 2}(\Omega, 0) \subset W^{2 m \cdot 2}(\Omega)$. The estimate $(3.2)$ yields

$$
\left\|u_{\downarrow}-I_{h} u_{g} ; W^{m, 2}(\Omega)\right\| \leqq C h^{m}\left\|\nabla_{2 m} u ; L^{2}(\Omega)\right\| \leqq C h^{m}\left\|u ; V^{2 m, 2}(\Omega, 0)\right\| \leqq C\left\|g ; L^{2}(\Omega)\right\| .
$$


Therefore we get

$$
\left\|u-P_{h} u ; L^{2}(\Omega)\right\|=C h-\operatorname{Im} \lambda_{-}+N / 2-\varepsilon\left\|f ; L^{2}(\Omega)\right\| \text { for }-\operatorname{Im} \lambda_{+}+N / 2<0 .
$$

For symmetric bilinear forms we have

$$
\left\|u-P_{h} u ; L^{2}(\Omega)\right\| \leqq C h^{2}\left(-\operatorname{Im} \lambda_{-}+N / 2-m-\varepsilon\left\|f ; L^{2}(\Omega)\right\| .\right.
$$

3.4 Error estimates in $L^{p}(\Omega), 2 \leqq p \leqq \infty$. We have seen in Subsection 3.3 that the Aubin-Nitsche trick yields very simply error estimates in $L^{2}(\Omega)$. In order to get results in $L^{p}(\Omega)$ for $2 \leqq p \leqq \infty$, we need more complicated investigations.

Theorem 6: Assume that the suppositions of Theorem 5 are satisfied, that $(\mathrm{R})$ is valid for $a^{*}(\cdot, \cdot)$, that the right-hand side $f$ of $(1.10)$ lies in $L^{p}(\Omega), 2 \leqq p \leqq \infty, \operatorname{Im} \lambda_{-}$ $<N / p$, that $S_{h} \subset W^{m, p}(\Omega)$ for $p<\infty$ and that $S_{h} \subset L^{\infty}(\Omega)$ for $p=\infty$. Then the finiteelement solution $P_{h} u \in S_{h}$ approximates the solution $u$ of (1.10) in the sense

$$
\left\|u-P_{h} u, L^{p}(\Omega)\right\| \leqq C \begin{cases}h^{-\operatorname{Im} \lambda_{-}+N / p-\varepsilon}\left\|f ; L^{p}(\Omega)\right\| & \text { if }-\operatorname{Im} \lambda^{+}+N / p<0, \\ h^{-\operatorname{Im} \lambda_{-}+\operatorname{Im} \lambda_{+}-\varepsilon}\left\|f ; L^{p}(\Omega)\right\| & \text { if }-\operatorname{Im} \lambda^{+}+N / p \geqq 0\end{cases}
$$

provided $m \geqq N / 2$, and $\operatorname{Im} \lambda_{-}+m<0$ and $2 \leqq p \leqq \infty$, or $\operatorname{Im} \lambda_{-}+m \geqq 0$ and $2 \leqq p<N /\left(\operatorname{Im} \lambda_{-}+m\right)$;

$$
\begin{aligned}
& \left\|u-P_{h} u ; L^{p}(\Omega)\right\| \\
& \leqq C \begin{cases}h^{-\operatorname{Im} \lambda_{-}+N / p-\varepsilon}\left\|f ; L^{p}(\Omega)\right\| & \text { if }-\operatorname{Im} \lambda_{+}+\operatorname{Im} \lambda_{-}+m<0, \\
h^{-2 \operatorname{Im} \lambda_{-}+N / p+\operatorname{Im} \lambda_{+}-m-\varepsilon}\left\|f ; L^{p}(\Omega)\right\| & \text { if }-\operatorname{Im} \lambda_{+}+\operatorname{Im} \lambda_{-}+m \geqq 0\end{cases}
\end{aligned}
$$

provided $\operatorname{Im} \lambda_{-}+m \geqq 0$ and $N /\left(\operatorname{Im} \lambda_{-}+m\right) \leqq p \leqq \infty$, where $\varepsilon>0$ is a small real number.

Proof: (i) We remark that if $m<N / 2$, then it follows from (3.6) that $\operatorname{Im} \lambda_{-}+m$ $>N / 2-m>0$. Therefore all cases are covered by the above assumptions.

(ii) We first consider the case $m \geqq N / 2, \operatorname{Im} \lambda_{-}+m<0,2<p<\infty$. We want to use Lemma 5 for $X=L^{p}(\Omega), X_{1}=W^{m, p}(\Omega)$ and $X_{2}=W^{m, q}(\Omega)$, where $1 / p+1 / q$ $=1$. Let us verify the assumptions of Lemma 5 . Since $N / p>N / 2-m$, the classical imbedding theorem yields that $V \subset W^{m \cdot 2}(\Omega) \subset L^{p}(\Omega)$. Since $\operatorname{Im} \lambda_{-}+m<0$, Lemma 2 vields that $u \in V^{2 m} \cdot p\left(\Omega, \operatorname{Im} \lambda_{-}-N / p+2 m+\varepsilon\right) \subset V^{m, p}\left(\Omega, \operatorname{Im} \lambda_{-}-N / p+m+\varepsilon\right)$ $\subset V^{m, p}(\Omega, 0) \subset W^{m, p}(\Omega)$. Since $N / p-m<\operatorname{Im} \lambda_{+}$and $\operatorname{Im} \lambda_{-}<N / p$, we finally get from Lemma 2 that $u_{g} \in W^{m . q}(\Omega)$ for $g \in L^{q}(\Omega)$. Therefore Lemma 8 is applicable and we have

$$
\left\|u-P_{h} u ; L^{p}(\Omega)\right\| \leqq C\left\|u-P_{h} u ; W^{m, p}(\Omega)\right\| \sup _{0 \neq g \in L^{q}(\Omega)} \frac{\left\|u_{g}-I_{h} u_{g} ; W^{m, q}(\Omega)\right\|}{\left\|g, L^{q}(\Omega)\right\|} .
$$

Let us estimate the first factor. It follows from (3.2) and (3.4) that

$$
\begin{aligned}
& \mid \text { i } u-P_{h} u ; W^{m, p}(\Omega) \| \\
& \leqq\left\|u-I_{h} u ; W^{m, p}(\Omega)\right\|+\left\|I_{h} u-P_{h} u ; W^{m, p}(\Omega)\right\| \\
& \leqq C\left(\left\|u-I_{h} u ; W^{m, p}(U(h))\right\|+\left\|u-I_{h} u ; W^{m, p}(\Omega \backslash U(h))\right\|\right. \\
& \left.+h^{N / p-N / 2}\left\|I_{h} u-P_{h} u ; W^{m, 2}(\Omega)\right\|\right) \\
& \leqq C\left(\left\|\nabla_{m} u ; L^{p}(U(h))\right\|+h^{m} \| \nabla_{2 m} u ; L^{p}(\Omega) \backslash U(h)\right) \\
& \left.+h^{N / p-N / 2}\left\|I_{h} u-u ; W^{m, 2}(\Omega)\right\|+h^{N / p-N / 2}\left\|u-P_{h} u ; W^{m, 2}(\Omega)\right\|\right) \text {. }
\end{aligned}
$$


Using Remark 2 and the relation (3.1) we have

and

$$
\begin{aligned}
& \left\|\nabla_{m} u ; L^{p}(U(h))\right\|^{p}=\sum_{|x|=m^{-}} \int_{U(h)} r^{p\left(\operatorname{Im} \lambda_{-}-N / p+m+\varepsilon\right)-p\left(\operatorname{Im} \lambda_{-}-N / p+m+\varepsilon\right)}\left|D^{x} u\right|^{p} d x \\
& \leqq c h^{-p\left(\operatorname{Im} \iota_{-}-N / p+m+\varepsilon\right)}\left\|u ; V^{2 m, p}\left(\Omega, \operatorname{Im} \lambda_{-}-N / p+2 m+\varepsilon\right)\right\|^{p}
\end{aligned}
$$

$$
\begin{aligned}
& \left\|\nabla_{2 m} u ; L^{p}(\Omega \backslash U(h))\right\|^{p} \\
& =\sum_{|\alpha|=2 m} \int_{(\Omega) \backslash U(h)} 1 h^{p\left(\operatorname{Im} \lambda_{-}-V / p-2 m+\varepsilon\right)-p\left(\operatorname{Im} \lambda_{-}-N / p+2 m+\varepsilon\right)}\left|D^{a} u\right|^{p} d x \\
& \leqq C h^{\left.-p(\operatorname{Im}\rangle_{-}-N / p+2 m+\varepsilon\right)}\left\|u ; V^{2 m, p}\left(\Omega, \operatorname{Im} \lambda_{-}-N / p+2 m+\varepsilon\right)\right\|^{p} .
\end{aligned}
$$

Therefore we get

$$
\begin{aligned}
\| u & -P_{h} u ; W^{m, p}(\Omega) \| \\
\leqq & C\left(h^{\left.-\operatorname{Im} \dot{\lambda}_{-}+N / p-m-\varepsilon\right)}\left\|u ; V^{2 m, p}\left(\Omega, \operatorname{Im} \lambda_{-}-N / p+2 m+\varepsilon\right)\right\|\right. \\
& \left.+h^{N / p-N / 2-\operatorname{Im} \lambda_{--T} / 2-m-\varepsilon}\left\|f ; L^{2}(\Omega)\right\|\right) \\
\leqq & C h^{-\operatorname{Im} \lambda_{-}+N / p-m-\varepsilon}\left\|f ; L^{p}(\Omega)\right\| .
\end{aligned}
$$

We now estimate s're second factor of (3.13), having in mind that $\operatorname{Im} \mu_{-}-N / q$ $+m+\varepsilon<0$, but $\operatorname{Im} \mu_{-}-N / q+2 m=-\operatorname{Im} \lambda_{+}+N / p \geqq 0$. Let us start with the case that $-\operatorname{Im} \lambda_{+}+N / p \geqq 0$. The estimate (3.2) and Remark 2 yield

$$
\begin{aligned}
& \left\|u_{g}-I_{h} u_{g} ; W^{m, q}(\Omega)\right\|^{q} \\
= & \left\|u_{g}-I_{h} u_{g} ; W^{m, q}(U(h))\right\|^{q}+\left\|u_{g}-I_{h} u_{g} ; W^{m, q}(\Omega \backslash U(h))\right\|^{q} \\
\leqq & C\left(\left\|\nabla_{m} u_{g} ; L^{q}(U(h))\right\|^{q}+h^{m q}\left\|\nabla_{2 m} u_{g} ; L^{q}(\Omega \backslash U(h))\right\|^{q}\right) \\
= & C\left(\sum_{|\alpha|=m} \int_{U(h)} r^{q\left(\operatorname{Im} \mu_{-}-N / q+m+\varepsilon\right)-q\left(\operatorname{Im} \mu_{-}-N / q+m+\varepsilon\right)}\left|D^{a} u_{g}\right|^{q} d x\right. \\
& +h^{m q} \| \nabla_{2 m} u_{g} ; L^{q}\left(\Omega \backslash U(h): \|^{q}\right) \\
\leqq & C\left(h^{q\left(-\operatorname{Im} \mu_{-}+N / q-m-s\right)}\left\|u_{g} ; V^{2 m, q}\left(\Omega, \operatorname{Im} \mu_{-}-N / q+2 m+\varepsilon\right)\right\|\right. \\
& \left.+h^{m q}\left\|\Gamma_{2 m} u_{g} ; L^{q}(\Omega \backslash U(h))\right\|^{q}\right) .
\end{aligned}
$$

An estimate similar to (3.15) implies

$$
\begin{aligned}
\left\|u_{g}-I_{h} u_{g} ; W^{m, q}(\Omega)\right\| & \leqq C h^{-\left(\operatorname{Im} \mu_{-}-N / q+2 m+\varepsilon\right)}\left\|g ; L^{q}(\Omega)\right\| \\
& =C h^{\operatorname{Im} \lambda_{+}-N / p+m-\varepsilon}\left\|g ; L^{q}(\Omega)\right\| .
\end{aligned}
$$

We now assume that $\operatorname{Im} \mu_{-}-N / q+2 m=-\operatorname{Im} \lambda_{+}+N / p<0$. Since $\operatorname{Im} \lambda_{-}$ $<N / p$, we have that $N / q-2 m<\operatorname{Im} \mu_{+}$, and Remark 2 yields that $u_{g} \in V^{2 m, q}(\Omega, 0)$ $\subset W^{2 m, q}(\Omega)$ and

$$
\left\|u_{g}-I_{h} u_{g} ; W^{m, q}(\Omega)\right\| \leqq C h^{m}\left\|g ; L^{q}(\Omega)\right\| .
$$

The inequalities (3.13), (3.16) -(3.18) imply the estimate (3.11).

(iii) Let $\operatorname{Im} \lambda_{-}+m \geqq 0$ and $2 \leqq p<N /\left(\operatorname{Im} \lambda_{-}+n\right)$. Then we have again $N / p>N / 2-m, u \in V^{2 m . p}\left(\Omega, \operatorname{Im} \lambda_{-}-N / p+2 m+\varepsilon\right) \subset V^{m, p}\left(\Omega, \operatorname{Im} \lambda_{-}-N / p+m\right.$ $+\varepsilon) \subset V^{m, p}(\Omega, 0) \subset W^{m, p}(\Omega)^{-}$and $\operatorname{Im} \lambda_{-}-N / p+2 m>N / 2-N / p \geqq 0$. Therefore we can repeat all estimates of the case (ii). 
(iv) Let $\operatorname{Im} \lambda_{-}+m \geqq 0, N /\left(\operatorname{Im} \lambda_{-}+m\right) \leqq p<\infty$. We have

$$
\left\|u-P_{h} ; L^{p}(\Omega)\right\| \leqq\left\|u-I_{h} u ; L^{p}(\Omega)\right\|+\left\|I_{h} u-P_{h} u ; L^{p}(\Omega)\right\| .
$$

We estimate the first term of the right-hand side using (3.2):

$$
\begin{aligned}
\left\|u-I_{h} u ; L^{p}(\Omega)\right\|^{p} & \leqq C\left\|u ; L^{p}(\Omega)\right\|^{p} \\
& =C\left\|u ; L^{p}(U(h))\right\|^{p}+C\left\|u ; L^{p}(\Omega \backslash U(h))\right\|^{p} .
\end{aligned}
$$

Since $\operatorname{Im} \lambda_{-}-N / p+\varepsilon<0$ and $\operatorname{Im} \lambda_{-}-N / p+2 m+\varepsilon>0$ if $\varepsilon$ is sufficiently small and $u \in V^{2 m, p}\left(\Omega, \operatorname{Im} \lambda_{-}-N / p+2 m+\varepsilon\right) \subset L^{p}\left(\Omega, \operatorname{Im} \lambda_{-}-N / p+\varepsilon\right)$, we get $\left\|u ; L^{p}(U(h))\right\|,\left\|u ; L^{p}(\Omega \backslash U(h))\right\| \leqq C h^{-\operatorname{Im} \lambda_{-}+N / p-\varepsilon}\left\|f ; L^{p}(\Omega)\right\|$ similar to (3.14) and (3.15), and therefore

$$
\left\|u-I_{h} u ; L^{p}(\Omega)\right\| \leqq C h^{-\operatorname{Im} \lambda_{-}+N / p-\varepsilon}\left\|f ; L^{p}(\Omega)\right\| .
$$

Using the inequality (3.4) for $q_{1}=N /\left(\operatorname{Im} \lambda_{-}+m+\delta\right)$, where $\delta>0$ is sufficiently small, we get for the second term of (3.19)

$$
\left\|I_{h} u-P_{h} u ; L^{p}(\Omega)\right\| \leqq C h^{N / p-\left(\operatorname{Im} \lambda_{-}+m+\delta\right)}\left\|I_{h} u-P_{h} u ; L^{N_{/}\left(\operatorname{Im} \lambda_{-}+m+\delta\right)}(\Omega)\right\| .
$$

Since $q_{1}<N /\left(\operatorname{Im} \lambda_{-}+m\right)$, we can apply estimate (3.20) and get

$$
\left\|I_{h} u-u ; L^{N /\left(\operatorname{Im} \lambda_{-}+m+\delta\right)}(\Omega)\right\| \leqq C h^{m+\delta-\varepsilon}\left\|f ; L^{N /\left(\operatorname{Im} \lambda_{-}+m+\delta\right)}(\Omega)\right\| .
$$

From (3.11) follows

$$
\begin{aligned}
& \left\|\dot{u}-P_{h} u ; L^{N /\left(\operatorname{Im} \lambda_{-}+m+\delta\right)}(\Omega)\right\| \\
& \leqq C \begin{cases}h^{m+\delta-\varepsilon}\left\|f ; L^{N /\left(\operatorname{Im} \lambda_{-}+m+\delta\right.}(\Omega)\right\| & \text { for }-\operatorname{Im} \lambda_{+}+\operatorname{Im} \lambda_{-}+m<0, \\
h^{-\operatorname{Im} \lambda_{-}+\operatorname{Im} \lambda_{+}-\varepsilon}\left\|f ; L^{N /\left(\operatorname{Im} \lambda_{-}+m+\delta\right)}(\Omega)\right\| & \text { for }-\operatorname{Im} \lambda_{+}+\operatorname{Im} \lambda_{-}+m \geqq 0 .\end{cases}
\end{aligned}
$$

Since $m<-\operatorname{Im} \lambda_{-}+N / p$, we get

$$
\begin{aligned}
& \left\|I_{h} u-P_{h} u ; L^{p}(\Omega)\right\| \\
& \leqq C \begin{cases}h^{N / p-\operatorname{Im} \lambda_{-}-\bullet}\left\|f ; L^{p}(\Omega)\right\| & \text { if }-\operatorname{Im} \lambda_{+}+\operatorname{Im} \lambda_{-}+m<0, \\
h^{N / p-2 \operatorname{Im} \lambda_{-}+\operatorname{Im} \lambda_{+}-m-\varepsilon-\delta}\left\|f ; L^{p}(\Omega)\right\| & \text { if }-\operatorname{Im} \lambda_{+}+\operatorname{Im} \lambda_{-}+m \geqq 0 .\end{cases}
\end{aligned}
$$

Since here $-\operatorname{Im} \lambda_{-}+N / p \geqq-2 \operatorname{Im} \lambda_{-}+N / p+\operatorname{Im} \lambda_{+}-m$ in the second case, we get from (3.20), (3.21) the assertion (3.12).

(v) At last we consider the case $p=\infty$. Here $\operatorname{Im} \lambda_{-}<0$. Let $q_{1}=N /\left(\operatorname{Im} \lambda_{-}\right.$ $+m+\delta$ ) as before, where $\delta>0$ is so small that $\operatorname{Im} \lambda_{-}+\delta<0$. We take an $\varepsilon>0$ such that $\operatorname{Im} \lambda_{-}-N / q_{1}+2 m+\varepsilon=m-\delta+\varepsilon \geqq 0$ and $\operatorname{Im} \lambda_{-}-N / q_{1}+m+\varepsilon$ $=-\delta+\varepsilon<0$. We have that $N / q_{1}<m$ and therefore it follows from Remark 2 and the classical imbedding theorem that $u \in V^{2 m, q}\left(\Omega, \operatorname{Im} \lambda_{-}-N / q_{1}+2 m+\varepsilon\right)$ $\subset V^{m, q_{1}}\left(\Omega, \operatorname{Im} \lambda_{-}-N / q_{1}+m+\varepsilon\right) \subset V^{m, q_{1}}(\Omega, 0) \subset W^{m, q_{1}}(\Omega) \subset L^{\infty}(\Omega)$. Using the estimates (3.3) and (3.4) we have

$$
\begin{aligned}
& \left\|u-P_{h} u ; L^{\infty}(\Omega)\right\| \\
& \leqq\left\|u-I_{h} u ; L^{\infty}(\Omega)\right\|+\left\|I_{h} u-P_{h} u ; L^{\infty}(\Omega)\right\| \\
& \leqq\left\|u-I_{h} u ; L^{\infty}(U(h))\right\|+\left\|u-I_{h} u ; L^{\infty}(\Omega \backslash U(h))\right\| \\
& +\left\|I_{h} u-P_{h} u ; L^{\infty}(\Omega)\right\| \\
& \leqq C\left(h^{m-N / q_{1}}\left\|\nabla_{m} u ; L^{q_{1}}(U(h))\right\|+h^{2 m-N / q_{1}}\left\|\nabla_{2 m} u ; L^{q_{1}}(\Omega \backslash U(h))\right\|\right. \\
& \left.+h^{-N / p}\left\|I_{h} u-P_{h} u ; L^{p}(\Omega)\right\|\right),
\end{aligned}
$$


where $p$ is so large that $N / p<\varepsilon$ and $p>2$. Since $\operatorname{Im} \lambda_{-}-N / p+2 m+\varepsilon>0$, it follows from Remark 2 that $u \in V^{2 m, p}\left(\Omega, \operatorname{Im} \lambda_{-}-N / p+2 m+\varepsilon\right) \subset L^{p}\left(\Omega, \operatorname{Im} \lambda_{-}\right.$ $-N / p+\varepsilon)$. The same ideas as were used in the estimates (3.14), (3.15) yield

$$
\left\|u-I_{h} u ; L^{\infty}(\Omega)\right\| \leqq C h^{-\operatorname{Im} \lambda_{-}-\varepsilon}\left\|f ; L^{q_{1}}(\Omega)\right\|
$$

and

$$
\left\|u-I_{h} u ; L^{p}(\Omega)\right\| \leqq C h^{-\operatorname{Im} \lambda_{-}+N / p-\varepsilon}\left\|f ; L^{p}(\Omega)\right\| .
$$

Moreover we demand that $p$ is so large that $-\operatorname{Im} \lambda_{+}+N / p<0$ provided $-\operatorname{Im} \lambda_{+}$ $<0$. We now apply the estimates (3.11) and (3.12) and get

$$
\begin{aligned}
& h^{-N / p}\left\|u-P_{h} u ; L^{p}(\Omega)\right\| \\
& \leqq C \begin{cases}h^{-\operatorname{Im} \lambda_{-}-\varepsilon}\left\|f ; L^{p}(\Omega)\right\| & \text { if }-\operatorname{Im} \lambda_{+}<0, \\
h^{-\operatorname{Im} \lambda_{-}+\operatorname{Im} \lambda_{+}-N / p-\varepsilon}\left\|f ; L^{p}(\Omega)\right\| & \text { if }-\operatorname{Im} \lambda_{+} \geqq 0\end{cases}
\end{aligned}
$$

provided $m \geqq N / 2$ and $\operatorname{Im} \lambda_{-}+m<0$ or $\operatorname{Im} \lambda_{-}+m \geqq 0$ and $2 \leqq p$ $<N /\left(\operatorname{Im} \lambda_{-}+m\right)$;

$$
\begin{aligned}
& h^{-N / p}\left\|u-P_{h} u ; L^{p}(\Omega)\right\| \\
& \leqq C \begin{cases}h^{-\operatorname{Im} \lambda_{-}-\varepsilon}\left\|f ; L^{p}(\Omega)\right\| & \text { if }-\operatorname{Im} \lambda_{+}+\operatorname{Im} \lambda_{-}+m<0, \\
h^{-2 \operatorname{Im} \lambda_{-}+\operatorname{Im} \lambda_{+}-m-\varepsilon}\left\|f ; L^{p}(\Omega)\right\| & \text { if }-\operatorname{Im} \lambda_{+}+\operatorname{Im} \lambda_{-}+m \geqq 0\end{cases}
\end{aligned}
$$

provided $\operatorname{Im} \lambda_{-}+m \geqq 0$ and $N /\left(\operatorname{Im} \lambda_{-}+m\right) \leqq p<\infty$. Sinće $-\operatorname{Im} \lambda_{-}+\operatorname{Im} \lambda_{+}$ $=-\operatorname{Im} \lambda_{-}$in the second case of (3.24) and $-2 \operatorname{Im} \lambda_{-}+\operatorname{Im} \lambda_{+}-m \leqq-\operatorname{Im} \lambda_{-}$in the second case of (3.25), we get the inequalities (3.11) and (3.12) also for $p=\infty$, inserting the estimates $(3.22)-(3.25)$ in the above estimate of $u-P_{h} u$ in $L^{\infty}(\Omega)$

Example 3: Let us consider the plane Dirichlet problem for the Laplace operator of Example 1. Assume there is only the corner point $0 \in \partial \Omega$ with an angle $\omega_{0}>\pi$. The corresponding bilinear form $a(\cdot, \cdot)$ is symmetric and therefore we have $\mu_{-}=\lambda_{-}$and $\mu_{+}=\lambda_{+}$. Since $\mu_{-}=\bar{\lambda}_{+}$ $+\mathrm{i}(N-2 m)=\bar{\lambda}_{+}=\lambda_{-}$, we get the well-known result $\operatorname{Im} \lambda_{+}=-\operatorname{Im} \lambda_{-}=\pi / \omega_{0}$ in our case.

We consider the case $p=\infty$. Since $\operatorname{Im} \lambda_{-}+m=-\pi / \omega_{0}+1>0$ and $-\operatorname{Im} \lambda_{+}+\operatorname{Im} \lambda_{-}$ $+m=-2 \pi / \omega_{0}+1<0$ for $\omega_{0}<2 \pi$, the first estimate of (3.12) implies $\left\|u-P_{h} u ; L^{\infty}(\Omega)\right\|$ $\leqq C h^{\pi / \omega_{\sigma}-\varepsilon} \| f ; L^{\infty}\left[(\Omega) \|\right.$. If $\omega_{0}=2 \pi$, the second estimate of $(3.12)$ yields $\left\|u-P_{h} u ; L^{\infty}(\Omega)\right\|$ $\leqq C h^{1 / 2-\varepsilon}\left\|f ; L^{\infty}(\Omega)\right\|$.

Example 4: We investigate the plane Dirichlet problem for the biharmonic operator, i.e. $\Delta^{2} u=f$ in $\Omega, u=\partial u / \partial u=0$ on $\partial \Omega$. Let $\Omega$ be a domain with the single corner point 0 with an angle $\omega_{0}>126^{\circ}$. The corresponding bilinear form $a(\cdot, \cdot)$ is symmetric and consequently we have $\mu_{-}=\bar{\lambda}_{+}+\mathrm{i}(N-2 m)=\bar{\lambda}_{+}+\mathrm{i}(-2)=\lambda_{-}$and $\operatorname{Im} \lambda_{+}=-\operatorname{Im} \lambda_{-}-2$. H. MELzeR and R. RANNACHER [13] have calculated the numbers $\operatorname{Im} \lambda_{-}$for ongles $\omega_{0} \in(0,2 \pi]$. In our case we have then $-3 \leqq \operatorname{Im} \lambda_{-} \leqq-1.5$ and consequently $-0.5 \leqq \operatorname{Im} \lambda_{+} \leqq 1$.

Let us consider the case $p=\infty$. We divide it into two subcases.

$1^{\circ} \operatorname{Im} \lambda_{-}+m<0$, which means $\omega_{0}<3 \pi / 2$. Consequently, $-\operatorname{Im} \lambda_{+}=\operatorname{Im} \lambda_{-}+m<0$, and the first estimate of (3.11) yields $\left\|u-P_{h} u ; L^{\infty}(\Omega)\right\| \leqq C h-\operatorname{Im} \lambda_{-}-\varepsilon\left\|f ; L^{\infty}(\Omega)\right\|$ if $\omega_{0}<3 \pi / 2$.

$2^{\circ} \operatorname{Im} \lambda_{-}+m \geqq 0$, which means $\omega_{0}>3 \pi / 2$. Then $-\operatorname{Im} \lambda_{+}+\operatorname{Im} \lambda_{-}+m=2\left(\operatorname{Im} \lambda_{-}+m\right)$ $\geqq 0$, and the second estimate of (3.12) yields $\left\|u-P_{h} u ; L^{\infty}(\Omega)\right\| \leqq C h-3 \operatorname{Im} \lambda_{-}-4-\varepsilon\left\|f ; L^{\infty}(\Omega)\right\|$ if $\omega_{0} \geqq 3 \pi / 2$. Especially for $\omega_{0}=2 \pi$ we have $\left\|u-P_{h} u ; L^{\infty}(\Omega)\right\| \leqq C h^{1 / 2-\varepsilon}\left\|f ; L^{\infty}(\Omega)\right\|$. 


\section{Finite-element methods in domains with edges}

We pick up the threads of Subsection 3.1. We assume for simplicity that the domain $\Omega \subset \mathbb{R}^{N}, N \geqq 3$, has the only edge $\bar{M}$. We take an analogous partition of $\bar{\Omega}$ and introduce the set $U(h)$ in the same way as in (3.1). We deal with the family $\left\{S_{h}\right\}$ of finite-element spaces with the properties $1^{\circ}$ and $2^{\circ}$ of Subsection 3.1 .

4.1 Error estimates in $W^{m, 2}(\Omega)$. The quality of the error estimates depends essentially on the regularity of the solution $u$ of $(1.10)$. Therefore we first introduce the definition of a significantly singular edge, analogously to the Definition 2 of a significantly singular point. that

Definition 4: The edge $M$ is significantly singular if there is a point $z_{1} \in M$ such

$$
1-2 m<\operatorname{Im} \lambda_{-}\left(z_{1}\right),
$$

where $\lambda_{-}\left(z_{1}\right)$ was defined by $(2.4)$.

We denote $\operatorname{Im} \lambda_{-}=\sup \left\{\operatorname{Im} \lambda_{-}\left(z_{1}\right): z_{1} \in M\right\}=\operatorname{Im} \lambda_{-}\left(z_{0}\right)$ as in Subsection 2.4. If (4.1) is valid and the assumptions of Lemma $5^{\prime}$ are satisfied, then for every $f \in L^{2}(\Omega)$ the solution $u$ of $(1.10)$ is from $V^{2 m \cdot 2}\left(\Omega, \operatorname{Im} \lambda_{-}-1+2 m^{\prime}+\varepsilon\right)$, but $V^{2 m, 2}\left(\Omega, \operatorname{Im} \lambda_{-}\right.$ $-1+2 m+\varepsilon) \nsubseteq V^{2 m, 2}(\Omega, 0) \subset W^{2 m, 2}(\Omega)$.

Example 5: We consider the Dirichlet problem $\Delta u=-f$ in $\Omega, u=0$ on $\partial \Omega$, where $\Omega \subset \mathbb{R}^{3}$ is a rotation-symmetric domain with an edge with constant interior angle $3 \pi / 2$. Then $\operatorname{Im} \lambda_{-}$ $=-2 / 3$. The relation (4.1) is satisfied and $M I$ is significantly singular.

Theorem $7:$ Let $a(\cdot, \cdot)$ be a real bilinear form which satisfies the conditions of the Lax-Milgram Theorem (2.6) and (2.7). Assume that there is only one significantly singular edge $M$, that the line $\operatorname{Im} \lambda=1-m$ is free of eigenvalues of $\mathfrak{A}_{0}(z, \lambda)$ for $z \in M$ and that the property $(\mathrm{R})$ is valid. Let $\left\{S_{h}\right\}$ be a family of finite-element spaces with the properties $1^{\circ}$ and $2^{\circ}$. Then the finite-element solution $P_{h} u \in S_{h}$ approximates the solution $u$ of (1.10) in the sense

$$
\left\|u-P_{h} u ; W^{m, 2}(\dot{\Omega})\right\| \leqq C h^{-\operatorname{Im} \hat{i}_{-}-m+1-\varepsilon}\left\|f ; L^{2}(\Omega)\right\|
$$

provided $f \in L^{2}(\Omega)$.

Proof: Using the ideas of the proof of Theorem 5 and the estimate (2.10) we get the assertion

4.2 Error estimates in $L^{p}(\Omega), 2 \leqq p \leqq \infty$. The basic idea in Section 3 was to use the Aubin-Nitsche trick in order to get error estimates in $L^{p}(\Omega), 2 \leqq p \leqq \infty$. The inequality (3.10), the regularity of the weak solution $u$ of problem (1.10) and the regularity of the solution of the corresponding adjoint problem (see Definition 3 ) are the keys for these estimates. The regularity of the solution $u$ was discussed in Subsection 2.4. Let us now formulate some results about the regularity of the solution of the adjoint problem.

Lemma 9 [12: §5]: Let $\mathfrak{A}_{0}^{*}(z, \mu)$ be the operator which is determined by $a^{*}(\cdot, \cdot)$ and which is defined analogously to $\mathfrak{A}_{0}(z, \hat{\lambda})$ (cf. (2.1)). Then $\lambda_{0}(z)$ is an eigenvalue of $\mathfrak{A}_{0}(z, \lambda)$ iff $\mu_{0}(z)=\overline{\lambda_{0}(z)}+\mathrm{i}(2-2 m)$ is an eigenvalue of $\mathfrak{A}_{0} *(z, \mu)$.

Corollaries: (i) If the line $\operatorname{Im} \lambda=-\dot{m}+1$ is free of eigenvalues of $\mathfrak{A}_{0}(z, \lambda)$ for all $z \in M$, then it is free of eigenvalues of $\mathfrak{A}_{0}^{*}(z, \mu)$, too. (ii) The imaginary parts of the eigenvalues of $\mathfrak{A}_{0}\left(z, \lambda_{)}\right)$and $\mathfrak{A}_{0} *(z, \mu)$ are situated symmetrically to the point $-m+1$ (cf. Fig. 3). (iii) Let $\lambda_{-}=\lambda_{-}\left(z_{0}\right)$ be an eigenvalue of $\mathfrak{A}_{0}\left(z_{0}, \lambda\right)$ given by Definition 4 and 
$\lambda_{+}=\lambda_{+}\left(\tilde{z}_{0}\right)$ an eigenvalue of $\mathfrak{A}_{0}\left(\tilde{z}_{0}, \hat{\lambda}\right)$ for which $\operatorname{Im} \hat{\lambda}_{+}=\operatorname{Im} \hat{\lambda}_{+}\left(\bar{z}_{0}\right) \leqq \operatorname{Im} \lambda_{+}(z)$ for all $z \in M I$. The corresponding eigenvalues of the adjoint problem are $\mu_{-}=\bar{\lambda}_{+}+\mathrm{i}(2-2 m)$ and $\mu_{+}=\bar{\lambda}_{-}+\mathrm{i}(2-2 m)$, and consequently we have $\operatorname{Im} \mu_{-}=-\operatorname{Im} \lambda_{+}-2 m+2$ and $\operatorname{Im} \mu_{+}=-\operatorname{Im} \lambda_{-}-2 m+2$.

Theorem 8: Assume that the suppositions of Theorem 7 are satisfied and that the property (R) holds for $a^{*}(\cdot, \cdot)$, too, that the right-hand side of $(1.10)$ is from $L^{p}(\Omega)$, $2 \leqq p \leqq \infty$, that $S_{h} \subset W^{m, p}(\Omega)$ for $p<\infty$ and $S_{h} \subset L^{\infty}(\Omega)$ for $p=\infty$. Then the finite-element solution $P_{h} u \in S_{h}$ approximates the solution $u$ of $(1.10)$ in the following way: If one of the conditions
a) $m \geqq \frac{N}{2}, \operatorname{Im} \lambda_{-}+m<0,2 \leqq p<\infty$,
b) $m \geqq \frac{N}{2}, \operatorname{Im} \lambda_{-}+m \geqq 0,2 \leqq p<\frac{2}{\operatorname{Im} \lambda_{-}+m}$,
c) $m<\frac{N}{2}, \xi \leqq p<\frac{2}{\operatorname{Im} \lambda_{-}+m} \leqq \frac{2 N}{N-2 m}$,
d) $m<\frac{N}{2}, 2 \leqq p \leqq \frac{2 N}{N-2 m}<\frac{2}{\operatorname{Im} i .+m}$

is fulfilled, then

$$
\begin{aligned}
& \left\|u-P_{h} u ; L^{p}(\Omega)\right\| \\
& \leqq C \begin{cases}h^{N / p-N / 2-\operatorname{Im} \lambda_{-}+1-\varepsilon}\left\|f ; L^{p}(\Omega)\right\| & \text { if }-\operatorname{Im} \lambda_{+}+2 / p<0, \\
h^{N / p-N / 2-\operatorname{Im} \lambda_{-}+\operatorname{Im} \lambda_{+}-2 / p+1-\varepsilon} \| f ; L^{p}(\Omega) ! & \text { if }-\operatorname{Im} \lambda_{+}+2 / p \geqq 0 .\end{cases}
\end{aligned}
$$

If $2 /\left(\operatorname{Im} \lambda_{-}+m\right) \leqq p^{\prime}<\infty$ and one of the conditions
e) $m \geqq \frac{N}{2}, \operatorname{Im} \lambda_{-}+t m \geqq 0$,
f) $m<\frac{N}{2}, \frac{2}{\operatorname{Im} \lambda_{-}+m} \leqq \frac{2 N}{N-2 m}$

is fulfilled, then

$$
\begin{aligned}
& \left\|u-P_{h} u ; L^{p}(\Omega)\right\| \\
& \leqq C \begin{cases}h^{N / p-N / 2-\operatorname{Im} \lambda_{+}+1-\varepsilon} i f ; L^{p}(\Omega) \| & \text { if }-\operatorname{Im} \lambda_{+}+\operatorname{Im} \lambda_{-}+m<0, \\
h^{N / p-N / 2-2 \operatorname{Im} \lambda_{-}+\operatorname{Im} \lambda_{+}-m+1-\varepsilon}\left\|f ; L^{p}(\Omega)\right\| & \text { if } \operatorname{Im} \lambda_{-}-\operatorname{Im} \lambda_{+}+m \geqq 0 .\end{cases}
\end{aligned}
$$

If

$$
\text { g) } m<\frac{N}{2}, \frac{2 N}{N-2 m}<\frac{2}{\operatorname{Im} \lambda_{-}+m}, \quad \frac{2 N}{N-2 m} \leqq p<\infty \text {, }
$$

then

$$
\begin{aligned}
& \left\|u-P_{h} u ; L^{p}(\Omega)\right\| \\
& \leqq C \begin{cases}h^{N / p-N / 2-\operatorname{Im} \lambda_{-}+1,-\varepsilon}\left\|f ; L^{p}(\Omega)\right\| & \text { if }-\operatorname{Im} \lambda_{+}+1-2 m / N<0, \\
h^{N / p-N / 2-\operatorname{Im} \lambda_{-}+\operatorname{Im} \lambda_{+}+2 m / N-\varepsilon}\left\|f ; L^{p}(\Omega)\right\| & \text { if }-\operatorname{Im} \lambda_{+}+1-2 m / N \geqq 0,\end{cases}
\end{aligned}
$$

where $\varepsilon>0$ is a small real number. If $p=\infty$ and $N\left(\operatorname{Im} \lambda_{-}+m\right) / 2<m$, then the corresponding estimates are valid, too. 
Proof: Cases a) and b): It follows from the classical imbedding theorems that $V \subset W^{m \cdot 2}(\Omega) \subset L^{p}(\Omega)$ and from Remark 3 that $u \in V^{2 m, p}\left(\Omega, \operatorname{Im} \lambda_{-}-2 / p+2 m+\varepsilon\right)$ $\subset V^{\prime m} \cdot p\left(\Omega, \operatorname{Im} \lambda_{-}-2 / p+m+\varepsilon\right) \subset V^{m \cdot p}(\Omega, 0) \subset W^{m, p}(\Omega)$ for a sufficiently small $\varepsilon>0$. For the solution $u_{g}$ of $a^{*}\left(u_{g}, v\right)=(g, v)$, where $g \in L^{q}(\Omega), 1 / p+1 / q=1$, we have $u_{g} \in V^{2 m, q}\left(\Omega, \operatorname{Im} \mu_{-}-2 / q+2 m+\varepsilon\right) \subset V^{m, q}\left(\Omega, \operatorname{Im} \mu_{-}-2 / q+m+\varepsilon\right)$ $=V^{m, q}\left(\Omega,-\operatorname{Im} \lambda_{+}+2 / p-m+\varepsilon\right) \subset W^{m \cdot q}(\Omega)$ because $2 / p-m<\operatorname{Im} \lambda_{+}$. Therefore Lemma 8 is applicable for $X=L^{p}(\Omega), X_{1}=W^{m \cdot p}(\Omega)$ and $X_{2}=W^{m \cdot q}(\Omega)$ and we have

$$
\left\|u-P_{h} u: L^{p}(\Omega) ! \mid C\right\| u-P_{h} u ; W^{m, p}(\Omega) \| \sup _{0 \neq g \in L^{q}(\Omega)} \frac{\left\|u_{g}-I_{h} u_{g} ; W^{m, q}(\Omega)\right\|}{\left\|g ; L^{q}(\Omega)\right\|} .
$$

We estimate the first factor, using the inequalities (3.2) and (3.4):

$$
\begin{aligned}
\| u & -P_{h} u: W^{m, p}(\Omega) \| \\
\leqq & \left\|u-I_{h} u ; W^{m, p}(\Omega)\right\|+\left\|I_{h} u-P_{h} u ; W^{m, p}(\Omega)\right\| \\
\leqq & C\left(\left\|u-I_{h} u ; W^{m, p}(U(h))\right\|+\left\|u-I_{h} u ; W^{m, p}(\Omega \backslash U(h))\right\| .\right. \\
& \left.+h^{N / p-N / 2}\left\|\dot{I}_{h} u-P_{h} u ; W^{m, 2}(\Omega)\right\|\right) .
\end{aligned}
$$

Using the properties of $U(h)$ we get, analogously to the proof of Theorem 6 , that

$$
\left\|u-I_{h} u ; W^{m, p}(\Omega)\right\| \leqq C h^{-\operatorname{Im} \lambda+2 / p-m-\varepsilon}\left\|f ; L^{p}(\Omega)\right\|,
$$

having in mind the inequality (2.10). Further it follows from (4.2) that

$$
h^{N / p-N / 2}\left\|I_{h} u-P_{h} u ; W^{m, 2}(\Omega)\right\| \leqq C h^{N / p-N / 2-\operatorname{Im} \lambda_{-}-m+1-\varepsilon}\left\|f ; L^{2}(\Omega)\right\| .
$$

Since $-\operatorname{Im} \lambda_{-}+2 / p-m>N / p-N / 2-\operatorname{Im} \lambda_{-}-m+1$ we get

$$
\left\|u-P_{h} u ; W^{m, p}(\Omega)\right\| \leqq C h^{N / p-N / 2-\operatorname{Im} \lambda_{-}-m+1-\varepsilon} \forall f ; L^{p}(\Omega) \| .
$$

We now estimate the second factor of (4.6). The estimate (3.2) and Remark 3 yield

$$
\begin{aligned}
& \left\|u_{g}-I_{h} u_{g} ; W^{m, q}(\Omega)\right\| \\
& \leqq C \begin{cases}h^{m}\left\|g ; L^{q}(\Omega)\right\| & \text { if }-\operatorname{Im} \lambda_{+}+2 / p<0, \\
h^{\operatorname{Im} \lambda_{+}+m-2 / p-\varepsilon}\left\|g ; L^{q}(\Omega)\right\| & \text { if }-\operatorname{Im} \lambda_{+}+2 / p \geqq 0 .\end{cases}
\end{aligned}
$$

We have used that $\operatorname{Im} \mu_{-}-2 / q+2 m=-\operatorname{Im} \lambda_{+}+2 / p$. Inserting (4.7) and (4.8) into (4.6) we finally get (4.3).

Cases c) and d): The classical imbedding theorems imply that $V \subset W^{m, 2}(\Omega)$ $\subset L^{2 N /(N-2 m)}(\Omega) \subset L^{p}(\Omega)$. Further we have that $u \in W^{m, p}(\Omega)$ as in the cases a), b), and since $2 / p-m<\operatorname{Im} \lambda_{+}, u_{g}$ is from $W^{m, q}(\Omega), 1 / p+1 / q=1$. Therefore we again get the estimate (4.3).

Cases e) and f): Lemma 6 yields that $u \in L^{p}(\Omega)$. We have

$$
\left\|u-P_{h} u ; L^{p}(\Omega)\right\| \leqq\left\|u-I_{h} u ; L^{p}(\Omega)\right\|+\left\|I_{h} u-P_{h} u ; L^{\dot{p}}(\Omega)\right\| .
$$

It follows from (3.2), Remark 3 and the properties of $U(h)$ that

$$
\begin{aligned}
\left\|u-I_{h} u ; L^{p}(\Omega)\right\| & \leqq C\left\|u ; L^{p}(\Omega)\right\| \leqq C\left(\left\|u ; L^{p}(U(h))\right\|+\left\|u ; L^{p}(\Omega \backslash U(h))\right\|\right) \\
& \leqq C h^{-\operatorname{Im} \lambda_{-}+2 / p-\varepsilon}\left\|f ; L^{p}(\Omega)\right\|:
\end{aligned}
$$


Let $\delta>0$ be so small that $-\operatorname{Im} \lambda_{+}+\operatorname{Im} \lambda_{-}+m+\delta<0$, provided $-\operatorname{Im} \lambda_{+}+\operatorname{Im} \lambda_{-}$ $+m<0$. Since $2 /\left(\operatorname{Im} \lambda_{-}+m+\delta\right)<2 /\left(\operatorname{Im} \lambda_{-}+m\right)$, it follows from b) or c), using (3.4) for $q_{1}=2 /\left(\operatorname{Im} \lambda_{-}+m+\delta\right)$, that

$$
\begin{aligned}
& \left\|I_{h} u-P_{h} u ; L^{p}(\Omega)\right\| \\
& \leqq C \begin{cases}h^{N / p-N / 2-\operatorname{Im} \lambda_{-}+1-\varepsilon}\left\|f ; L^{p}(\Omega)\right\| & \text { if }-\operatorname{Im} \lambda_{+}+\operatorname{Im} \lambda_{-}+m<0, \\
h^{N / p-N / 2+\operatorname{Im} \lambda_{+}-2 \operatorname{Im} \lambda_{-}-m+1-\varepsilon}\left\|f ; L^{p}(\Omega)\right\| & \text { if }-\operatorname{Im} \lambda_{+}+\operatorname{Im} \lambda_{-}+m \geqq 0 .\end{cases}
\end{aligned}
$$

Since $2 / p-\operatorname{Im} \lambda_{-} \geqq N / p-N / 2-\operatorname{Im} \lambda_{-}+1$ and $2 / p-\operatorname{Im} \lambda_{-} \geqq N / p-N / 2$ $-2 \operatorname{Im} \lambda_{-}+\operatorname{Im} \lambda_{+}-m+1$ in the second case we get from (4.9) and (4.11) the estimate (4.4).

Case g): The inequalities (4.9) and (4.10) are again satisfied. We apply the inequality (3.4) for $q_{1}=2 N /(N-2 m)$ in order to estimate the second term of (4.9). We have $\left\|I_{h} u-P_{h} u ; L^{p}(\Omega)\right\| \leqq C h^{N / p-N / q_{1}}\left\|I_{h} u-P_{h} u ; L^{q_{1}}(\Omega)\right\|$. Using now the inequality (4.3) in the case d) for $q_{1}$ instead of $p$ we get

$$
\begin{aligned}
& \left\|I_{h} u-P_{h} u ; L^{q_{1}}(\Omega)\right\| \\
& \leqq\left\|I_{h} u-u ; L^{q_{1}}(\Omega)\right\| \\
& \quad+C \begin{cases}h^{-\operatorname{Im} \lambda_{-}-m+1-\varepsilon}\left\|f ; L^{q_{1}}(\Omega)\right\| & \text { if }-\operatorname{Im} \lambda_{+}+1-2 m / N<0, \\
h^{-\operatorname{Im} \lambda_{-}+\operatorname{Im} \lambda_{+}-m+2 m / N-\varepsilon}\left\|f ; L^{q_{2}}(\Omega)\right\| & \text { if }-\operatorname{Im} \lambda_{+}+1-2 m / N \geqq 0 .\end{cases}
\end{aligned}
$$

Since $-\operatorname{Im} \lambda_{-}+2 / q_{1} \geqq-\operatorname{Im} \lambda_{-}-m+1$ if $-\operatorname{Im} \lambda_{+}+1-2 m / N<0$ and $-\operatorname{Im} \lambda_{-}+2 / q_{1} \geqq-\operatorname{Im} \lambda_{-}+\operatorname{Im} \lambda_{+}-m+2 m / N$ if $-\operatorname{Im} \lambda_{+}+1-2 m / N \geqq 0$, the estimate (4.10) for $p=q_{1}$ implies

$$
\begin{aligned}
& \left\|I_{h} u-P_{h} u ; L^{q_{1}}(\Omega)\right\| \\
& \leqq c \begin{cases}h^{-\operatorname{Im} \lambda_{-}-m+1-\varepsilon}\left\|f ; L^{q_{1}}(\Omega)\right\| & \text { if }-\operatorname{Im} \lambda_{+}+1-2 m / N_{-}<0, \\
h^{-\operatorname{Im} \lambda_{-}+\operatorname{Im} \lambda_{+}-m+2 m / N-\varepsilon}\left\|f ; L^{q_{1}}(\Omega)\right\| & \text { if }-\operatorname{Im} \lambda_{+}+1-2 m / N \geqq 0 .\end{cases}
\end{aligned}
$$

The inequality (4.5) follows from (4.9), (4.10) and (4.12) because of $-\operatorname{Im} \lambda_{-}+2 / p$ $\geqq N / p-N / 2-\operatorname{Im} \lambda_{-}+1$ and $-\operatorname{mm} \lambda_{-}+2 / p \geqq N / p-N / 2-\operatorname{Im} \lambda_{-}+\operatorname{Im} \lambda_{+}$ $+2 m / N$ if $-\operatorname{Im} \lambda_{+}+1-2 m / N \geqq 0$.

Case $p=\infty$ : We assume that $N\left(\operatorname{Im} \lambda_{-}+m\right) / 2<m$ and that $\delta>0$ is such a small real number that $N\left(\operatorname{Im} \lambda_{-}+m+\delta\right) / 2<m$, too. Let $p_{0}=2 /\left(\operatorname{Im} \lambda_{-}+m+\delta\right)$. It follows from Remark 3 that $u \in V^{2 m, p_{0}}\left(\Omega, \operatorname{Im} \lambda_{-}-2 / p_{0}+2 m+\varepsilon\right) \subset V^{m, p_{0}}(\Omega$, $\left.\operatorname{Im} \lambda_{-}-2 / p_{0}+m+\varepsilon\right) \subset V^{m, p_{0}}(\Omega, 0) \subset W^{m, p_{0}}(\Omega) \subset L^{\infty}(\Omega)$. We have

$$
\left\|u-P_{h} u ; L^{\infty}(\Omega)\right\| \leqq\left\|u-I_{h} u ; L^{\infty}(\Omega)\right\|+\left\|I_{h} u-P_{h} u ; L^{\infty}(\Omega)\right\| .
$$

We estimate the first term of (4.13):

$$
\left\|u-I_{h} u ; L^{\infty}(\Omega)\right\|=\max \left(\left\|u-I_{h} u ; L^{\infty}(U(h))\right\|,\left\|u-I_{h} u ; L^{\infty}(\Omega \backslash U(h))\right\|\right) .
$$

We get from (3.3) and Remark 3

$$
\begin{aligned}
\left\|u-I_{h} u ; L^{\infty}(U(h))\right\| & \leqq C h^{m-N / p_{0}}\left\|\Gamma_{m} u ; L^{p_{0}}(U(h))\right\| \\
& \leqq C h^{m-N / p_{0}} h^{-\operatorname{Im} \lambda_{-}+2 / p_{0}-m-\varepsilon}\left\|u ; V_{,}^{m, p_{0}}\left(\Omega, \operatorname{Im} \lambda_{-}-2 / p_{0}+m+\varepsilon\right)\right\| \\
& \leqq C h^{-\operatorname{Im} \lambda_{-}-N / p_{0}+2 / p_{0}-\varepsilon}\left\|f ; L^{p_{0}}(\Omega)\right\| .
\end{aligned}
$$


Analogously we get

$$
\begin{aligned}
\left\|u-I_{h} u ; L^{\infty}(\Omega \backslash U(h))\right\| & \leqq C h^{2 m-N / p_{0}}\left\|\Gamma_{2 m} u ; L^{p_{0}}\left(\Omega \backslash U^{\top}(h)\right)\right\| \\
& \leqq C h^{-\operatorname{Im} \lambda-N / p_{0}+2 / p_{0}-\varepsilon}\left\|f ; L^{p_{0}}(\Omega)\right\| .
\end{aligned}
$$

Consequently we have

$$
\left\|u-I_{h} u ; L^{\infty}(\Omega)\right\| \leqq C h^{-\operatorname{Im} \lambda_{-}-N / p_{0}+2 / p_{0}-\varepsilon}\left\|f ; L^{p_{0}}(\Omega)\right\| .
$$

We now consider the second term of-(4.13). The estimate (3.4) yields for a very large $p$

$$
\left\|I_{h} u-P_{h} u ; L^{\infty}(\Omega)\right\| \leqq C h^{-N / p}\left\|I_{h} u-P_{h} u ; L^{p}(\Omega)\right\| .
$$

We estimate $\left\|u-P_{h} u ; L^{p}(\Omega)\right\|$ by (4.3), (4.4) or (4.5) and $\left\|u-I_{h} u ; L^{p}(\Omega)\right\|$ as before and get the assertion

Example 6: We consider the 3dimensional Dirichlet problem for the Laplacian:

$$
a(u, v)=\sum_{i=1}^{3} \int_{\Omega} \frac{\partial u}{\partial x_{i}} \frac{\partial v}{\partial x_{i}} d x=\int_{Q} f v d x \quad \forall v \in W_{0}^{1,2}(\Omega), j \in L^{\infty}(\Omega) .
$$

The weak solution $u \in W_{0}^{1,2}(\Omega)$ is uniquely determined. Let $\operatorname{Im} \lambda_{-}=-\pi / \omega_{0}, \omega_{0}>\pi$. We have $N\left(\operatorname{Im} \lambda_{-}+m\right) / 2=3\left(-\pi / \omega_{0}+1\right) / 2<3 / 4<m=1$. The assumptions of the case $\left.\mathrm{g}\right)$ are satisfied for $p=\infty$. Formula (4.5) yields the error estimate $\left\|u-P_{h} u ; L^{\infty}(\Omega)\right\|=O\left(h^{n / \omega_{0}-1 / 2-t}\right)$.

Example 7: We consider the 3dimensional Dirichlet problem for the biharmonic equation:

$$
a(u, v)=\sum_{i, j=1}^{3} \int_{\rho} \frac{\partial^{2} u}{\partial x_{i}^{2}} \frac{\partial^{2} v}{\partial x_{j}^{2}} d x=\int_{Q} f v d x \quad \forall v \in W_{0}^{2,2}(\Omega), f \in L^{\infty}(\Omega) .
$$

The weak solution $u \in W_{0}^{2,2}(\Omega)$ is uniquely determined. Let $\omega_{0}$ be the largest angle of $M$. The case a) is satisfied if $\operatorname{Im} \lambda_{-}+m<0$, which means $\sim 126^{\circ}<\omega_{0}<180^{\circ}$. The case e) is satisfied if $\operatorname{Im} \lambda+m \geqq 0$, which means $\omega_{0} \geqq \pi$ (see H. Melzer and R. RANnacher [13]). Consequently,

$$
\left\|u-P_{h} u ; L^{\infty}(\Omega)\right\|= \begin{cases}O\left(h-1 / 2-\operatorname{Im} \lambda_{-}-\ell\right) & \text { if } \sim 126^{\circ}<\omega_{0}<180^{\circ}, \\ O\left(h-3 \operatorname{Im} \lambda_{-}-9 / 2-\varepsilon\right) & \text { if } \omega_{0}>180^{\circ} .\end{cases}
$$

\section{REFERENCE}

[1] Burm, H.: Der Einfluß von Eckensingularitäten bei der numerischen Behandlung der biharmonischen Gleichung. Inauguraldissertation. Bonn: Universität 1981.

[2] Сьярлє, Ф.: Метод конечных әлементов для әллиптических задач. Москва: Изд-во Мир 1980.

[3] Doвrowolskis, M.: Numerical Approximation of Elliptic Intérface and Corner Problems. Habilitationsschrift. Bonn: Universität 1981.

[4] Edmunds, D. E., KufNer, A., and J. RAKosnik: Embedding of Sobolev Spaces with Weights of Power Type. Z. Anal. Anw. 4 (1985), 25-34.

[5] Кондратьев, В. А.: Краевые задачіг для әллиптическпх у̃равнениї в областях с коническими или угловыми точками. Тр. Моск. мат. об-а 16 (1967), 209-292.

[6] Кондратьев, В. А.: О гладкости решения задачи Дирихле для әллиптических уравнений второго порядка в кусочно-гладкой области. Дифф. ур-ия 6 (1970), 1831-1843.

[7] КондрАтьев, В. А.: Особенности решения задачи Дирихле для әллиптических уравненйи второго порядка в окрестности ребра. Дифф. ур-ия 13 (1977), 2026-2032.

[8] KUFNER, A., and A.-M. SÄNDIG: Some Applications of Weighted Sobolev Spaces (TeubnerTexte zur Mathematik: Vol. 100). Leipzig: B. G. Teubner Verlagsges. 1987. 
[9] МАзья, В. Г., и Б. А. ПлАмЕнЕвский: Об эллиптических краевых задачах в области с кусочно-гладкой границей. Тр. Симп. по Мех. спл. среды п родств. пробл. анализа 1971, том 1. Тбилиси: Мецниереба 1973, стр. 171-181.

[10] МАзья, В. Г.', и Б. А. ПлАмЕНЕВСКий: О коәффициентах в аспмптотике решений эллиптических краевых задач в областях с коническимп точкамп. Math. Nachr. 76 (1977), 29-60.

[11] МАзья, В. Г., и Б. А. ПЛАменевский: Оценки в $L_{p}$ и в классах Гельдера и принци максимума Миранди-Агмона для решенпй әлінптических краевых задач в областях с особыми точками границы. Math. Nachr. 81 (1978), 25-82.

[12] МАзья, В. Г., и Б. А. ПлАменЕвский: $L_{p}$-оценки в асимптотике решений әллиптических краевых задач в областях с ребрами. Тр. Моск. мат. об-а 37 (1978), 49-93.

[13] MELZER, H., and R. RANNACHER: Spannungskonzentrationen in Eckpunkten der Kirchhoffschen Platte. Bonn: Universität; Preprint 270, SFB 72, 1972.

[14] Rossmann, J.: Gewichtete Sobolev-Slobodezkij-Räume und Anwendungen auf elliptische Randwertaufgaben in Gebieten mit Kanten. Dissertation B. Rostock: Wilhelm-PieckUniversität 1988 .

Manuskripteingang: 07. 12. 1987

VERFASSER :

Prof. Dr. Anna-Margarete Sändig

Sektion Mathematik der Wilhelm-Pieck-Universität

Universitätsplatz

DDR-2500 Rostock 